\title{
Infrared conformality and bulk critical points: SU(2) with heavy adjoint quarks
}

\author{
Biagio Lucini, ${ }^{a}$ Agostino Patella, ${ }^{b, c}$ Antonio Rago ${ }^{c}$ and Enrico Rinaldi ${ }^{d}$ \\ ${ }^{a}$ College of Science, Swansea University, \\ Singleton Park, Swansea SA2 8PP, U.K. \\ ${ }^{b} \mathrm{PH}-\mathrm{TH}, \mathrm{CERN}$, \\ CH-1211 Geneva 23, Switzerland \\ ${ }^{c}$ School of Computing and Mathematics \& Centre for Mathematical Science, \\ Plymouth University, Plymouth PL4 8AA, U.K. \\ ${ }^{d}$ Higgs Centre for Theoretical Physics, SUPA, School of Physics and Astronomy, \\ University of Edinburgh, Edinburgh EH9 3JZ, U.K. \\ E-mail: b.lucini@swansea.ac.uk, agostino.patella@plymouth.ac.uk, \\ antonio.rago@plymouth.ac.uk, e.rinaldi@sms.ed.ac.uk
}

ABSTRACT: The lattice phase structure of a gauge theory can be a serious obstruction to Monte Carlo studies of its continuum behaviour. This issue is particularly delicate when numerical studies are performed to determine whether a theory is in a (near-)conformal phase. In this work we investigate the heavy mass limit of the $\mathrm{SU}(2)$ gauge theory with $N_{f}=2$ adjoint fermions and its lattice phase diagram, showing the presence of a critical point ending a line of first order bulk phase transition. The relevant gauge observables and the low-lying spectrum are monitored in the vicinity of the critical point with very good control over different systematic effects. The scaling properties of masses and susceptibilities open the possibility that the effective theory at criticality is a scalar theory in the universality class of the four-dimensional Gaussian model. This behaviour is clearly different from what is observed for $\mathrm{SU}(2)$ gauge theory with two dynamical adjoint fermions, whose (near-)conformal numerical signature is hence free from strong-coupling bulk effects.

Keywords: Lattice Gauge Field Theories, Beyond Standard Model, Technicolor and Composite Models

ARXIV EPRINT: 1309.1614 


\section{Contents}

1 Introduction 1

2 The model 2

3 Phase diagram 4

4 Spectrum measurements $\quad 8$

$\begin{array}{lll}5 & \text { Scaling properties } & 16\end{array}$

$\begin{array}{llr}6 & \text { Conclusions } & 17\end{array}$

\section{Introduction}

Despite the recent identification of a light Higgs boson at the LHC [1, 2], unveiling the mechanism of electroweak symmetry breaking is still an open problem in theoretical particle physics. Among the possibilities still on the table, the suggestion that a novel strong interaction displaying confinement $[3,4]$ and an anomalous dimension of the chiral condensate of order one [5-7] can be tested quantitatively with lattice simulations. In the quest for a theory that could realise concretely this scenario, several gauge theory models, with matter in the fundamental or in a two-index representation and various flavour and colour content have been studied with Monte Carlo methods (see [8-10] for recent reviews), with the space of parameters narrowed down using analytical input $[11,12]$.

A large anomalous dimension is expected to arise near the onset of the conformal window. Hence, to unambiguously ascertain conformality for a gauge theory, one needs to be able to robustly determine whether the theory has or is near to an infrared (IR) fixed point. Recent Monte Carlo studies have shown that the identification of IR fixed points on the lattice is not straightforward, since in numerical simulations the system has a finite size (while conformality would require infinite distances to be explored) and fermions have a finite mass (the conformal limit being for massless matter fields). In addition, lattice simulations are performed at fixed cutoff and for particular choices of the discretised action. Because of these unavoidable complications, there is wide consensus that, in lattice simulations aimed at ascertaining (near-)conformality of a gauge theory, evidences based on different approaches and different techniques need to be collected before one can exclude spurious lattice signatures being mistaken for genuine IR fixed points in the continuum. In the last few years, thanks to the joint effort of various groups, this programme has been carried out for $\mathrm{SU}(2)$ gauge theory with two adjoint Dirac fermions, for which the scaling of the spectrum [13-17] and the behaviour of the coupling constant under RG flow [18$21]$ strongly suggest IR conformality (see also [22-24] for earlier numerical investigations). 
More recent lattice studies of the theory are focused on controlling systematic effects [25-28] and on precise measurements of the anomalous dimension $[29,30]$. However the persistence of the IR fixed point in the continuum limit is still under investigation.

Lattice simulations in the (near-)conformal regime are made more difficult by the lack of experimental guidance. For this reason, all possible sources of uncertainties and ambiguities need to be carefully analysed. In this paper, we study the possibility that numerical indications of conformality in $\mathrm{SU}(2)$ gauge theory with two Dirac flavours in the adjoint representation are in fact due to the presence of a second order transition point in a system related to this theory. Namely we refer to a $\mathrm{SU}(2)$ gauge theory with mixed fundamental-adjoint action [31], which the aforementioned theory with dynamical fermions reduces to at leading non-trivial order in the hopping parameter expansion. This is a potential effect that has not been considered before in the literature. For its investigation, we will compare the scaling of the spectrum in the gluonic sector found for $\mathrm{SU}(2)$ with two adjoint Dirac fermions with the scaling of the pure gauge spectrum of the mixed action system near its quantum critical point. In doing so, we shed some light on the nature of this point, solving some controversies in the earlier literature [32-37].

The rest of the paper is organised as follows. After introducing the system we have investigated and elucidating its relationship with $\mathrm{SU}(2)$ with two adjoint Dirac fermions (section 2), in section 3 we present our results on the location of the critical point in the bare coupling plane. These are obtained from the study of plaquette differences in the two coexisting vacua along the first order phase transition line ending in it. We also show results for the susceptibility of the plaquette. Section 4 reports on our data for the spectrum, whose scaling properties are investigated in section 5. Our findings are then summarised in section 6. Preliminary results of our investigation have been reported in [38].

\section{The model}

In the Wilson discretisation of fermions, the lattice Dirac operator for a single fermion species of mass am (in lattice units) transforming in the representation $\mathrm{R}$ of the gauge group is given by

$$
\begin{aligned}
M_{\alpha \beta}(i j)=(a m+4 r) \delta_{i j} \delta_{\alpha \beta}-\frac{1}{2}\left[\left(r-\gamma_{\mu}\right)_{\alpha \beta} U_{\mu}^{\mathrm{R}}(i) \delta_{i, j-\hat{\mu}}\right. & \\
& \left.+\left(r+\gamma_{\mu}\right)_{\alpha \beta}\left(U_{\mu}^{\mathrm{R}}(i)\right)^{\dagger} \delta_{i, j+\hat{\mu}}\right],
\end{aligned}
$$

where $i$ and $j$ are lattice site indices, $\alpha$ and $\beta$ are Dirac indices, $\mu$ is an Euclidean direction and the $\gamma$ matrices are formulated in Euclidean space. $U_{\mu}^{\mathrm{R}}(i)$ is the link variable in the representation $\mathrm{R}$ of the gauge group $\mathrm{SU}\left(N_{c}\right)$. The path integral of a theory with $N_{f}$ flavours transforming in the representation $\mathrm{R}$ is then given by

$$
Z=\int\left(\mathcal{D} U_{\mu}(i)\right)\left(\operatorname{det} M\left(U_{\mu}\right)\right)^{N_{\mathrm{f}}} e^{-S_{\mathrm{F}}},
$$

where $S_{\mathrm{F}}$ is the gauge action, which for simplicity will be taken as the Wilson plaquette action:

$$
S_{\mathrm{F}}=\beta_{\text {fund }} \sum_{i, \mu>\nu}\left(1-\frac{1}{N_{c}} \operatorname{Re} \operatorname{Tr}_{F}\left(U_{\mu \nu}(i)\right)\right) .
$$


Here, $U_{\mu \nu}(i)$ is the plaquette in the $(\mu, \nu)$-plane from point $i$ and $\beta_{\text {fund }}=2 N_{c} / g^{2}$, with $g$ the bare coupling. The sum over all the points $i$ is done over the four-dimensional lattice $L^{3} \times T$. $\operatorname{Tr}_{F}$ is the trace operator defined in the fundamental representation of the $\mathrm{SU}\left(N_{c}\right)$ gauge group. For reasons that will be clear below, we call this the fundamental action (where fundamental refers to the fact that the plaquette is in the fundamental representation).

For large bare quark mass, $\operatorname{det} M$ can be expanded in powers of the hopping parameter $\kappa=[2(a m+4 r)]^{-1}$. At the leading non-trivial order, this gives

$$
Z=\int\left(\mathcal{D} U_{\mu}(i)\right) e^{-S_{\mathrm{eff}}}
$$

with

$$
S_{\mathrm{eff}}=S_{\mathrm{F}}+S_{\mathrm{R}}
$$

and (up to irrelevant constants)

$$
S_{R}=\tilde{\beta}_{\mathrm{R}} \sum_{i, \mu>\nu}\left(1-\frac{1}{d_{\mathrm{R}}} \operatorname{Re} \operatorname{Tr}_{\mathrm{R}}\left(U_{\mu \nu}(i)\right)\right),
$$

where $\operatorname{Tr}_{\mathrm{R}}$ is the trace in the representation $\mathrm{R}, d_{\mathrm{R}}$ the dimension of that representation and

$$
\tilde{\beta}_{\mathrm{R}}=8 \kappa^{4} d_{\mathrm{R}}\left(1+2 r^{2}-r^{4}\right)
$$

Eqs. (2.5)-(2.7) show that at high bare mass the dynamical system is approximated by a gauge system with a mixed action, i.e. with an action that, in addition to the fundamental Wilson term, has a coupling to the plaquette in the representation $\mathrm{R}$ governed by the mass of the fermions (assumed to be large). These variant actions are known to have a non-trivial phase structure in the plane of the couplings (see e.g. [39]).

If we specialise our derivation to the adjoint representation, eq. (2.5) becomes a particular case of the mixed fundamental-adjoint gauge action

$$
\begin{aligned}
S=\beta_{\text {fund }} \sum_{i, \mu>\nu}( & \left.1-\frac{1}{N_{c}} \operatorname{Re} \operatorname{Tr}_{F}\left(U_{\mu \nu}(i)\right)\right) \\
& +\beta_{\text {adj }} \sum_{i, \mu>\nu}\left(1-\frac{1}{N_{c}^{2}-1} \operatorname{Re} \operatorname{Tr}_{A}\left(U_{\mu \nu}(i)\right)\right),
\end{aligned}
$$

where $\operatorname{Tr}_{A}$ is the trace in the adjoint representation (whose dimension is $d_{A}=N_{c}^{2}-1$ ), related to the trace in the fundamental representation $\operatorname{Tr}_{F}$ by $\operatorname{Tr}_{A}(U)=\left|\operatorname{Tr}_{F}(U)\right|^{2}-1$. If we take $\mathrm{SU}(2)$ as the gauge group, the action (2.8) can be seen as a generalisation of the high bare mass regime of $\mathrm{SU}(2)$ gauge theory with two adjoint Dirac flavours.

For $N_{c}=2$, simulations with the mixed fundamental-adjoint action were already carried out in the early days of lattice gauge theories [31] and more recently in ref. [32, $33,35,36]$. A mixed-action study of the finite temperature transition for $\mathrm{SU}(3)$ was also considered in ref. [40]. These studies suggest the existence of a second order phase transition point in the bare coupling plane, which for $\mathrm{SU}(2)$ is attained at $\beta_{\text {adj }} \approx 1.25$ and $\beta_{\text {fund }} \approx$ 1.22. However, due to growing autocorrelation times, which makes it difficult to study the 


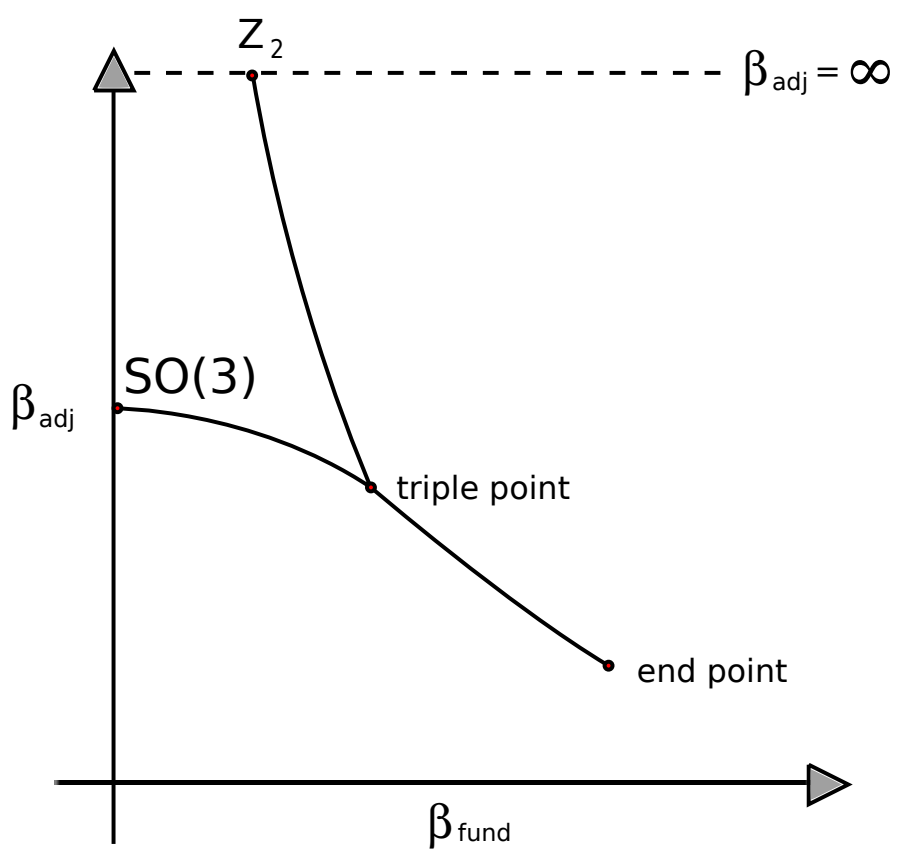

Figure 1. Sketch of the phase diagram for the lattice system defined by eq. (2.8). The lines of first order phase transitions are shown and are explained in section 3.

system in a neighbourhood of this critical point, this evidence has not been considered conclusive [37].

Due to the relationship between $\mathrm{SU}(2)$ with mixed fundamental-adjoint action and $\mathrm{SU}(2)$ with two Dirac flavours in the adjoint representation and to hints for nearconformality in the latter, it is important to analyse carefully the physics of the pure gauge system in its bare parameter space. In particular we explore the region around $\left(\beta_{\text {fund }}=1.22, \beta_{\text {adj }}=1.25\right)$ (see section 3 for details), to understand the signatures of a possible end-point on the physical spectrum and to compare the behaviour of masses near it with the scaling of masses in $\mathrm{SU}(2)$ with two adjoint Dirac flavours.

\section{Phase diagram}

From previous works on small lattices [31] and from analytical arguments [39], it was known that the lattice system described by eq. (2.8) had an interesting non-trivial phase diagram. A sketch of the phase diagram is presented in figure 1. The bare parameter space of the couplings $\left(\beta_{\text {fund }}, \beta_{\text {adj }}\right)$ contains two first order phase transition lines that belong to theories in different limits of the system: the $\mathbb{Z}_{2}$ gauge theory at $\beta_{\text {adj }}=\infty$ and the $\operatorname{SO}(3)$ gauge theory at $\beta_{\text {fund }}=0$. These two lines merge in a triple point at finite values of the couplings and continue as a single line toward the fundamental Wilson axis $\beta_{\text {adj }}=0$. This single line is thought to be a line of bulk first order transition points that ends around $\beta_{\text {adj }} \approx 1.25$. Due to the possibility of this end-point being of second order, it is necessary to carefully investigate the nature of the transition on large volume lattices and at high statistics, which is required by the large autocorrelation times of the system. For our Monte 


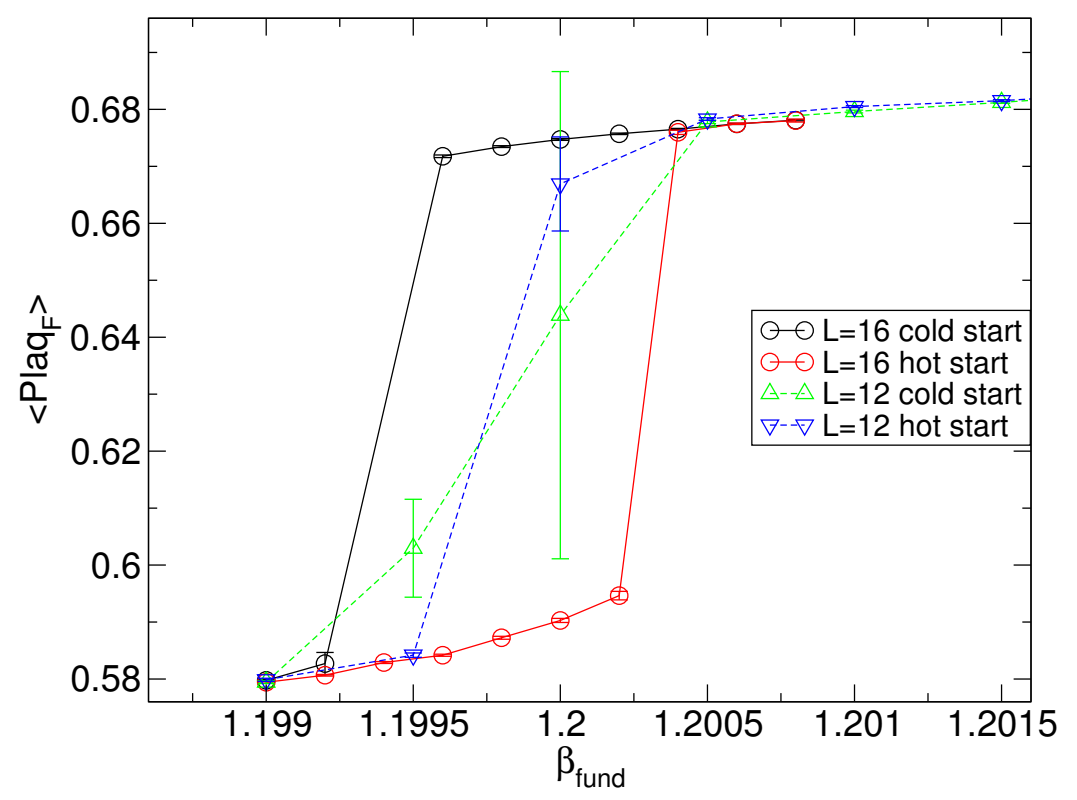

Figure 2. Fundamental plaquette expectation values for several $\beta_{\text {fund }}$ couplings at $\beta_{\text {adj }}=1.275$ on two different volumes $L=12,16$. The hysteresis cycle, clearly visible on the larger volume, is hard to identify on the smaller one. A similar consistent picture holds for the expectation values of the modulus of Polyakov loops.

Carlo simulations we employ a biased Metropolis algorithm that has been proven to have an heatbath-like efficiency [41, 42]. This algorithm helped us reduce autocorrelations with respect to the standard multi-hit Metropolis on which earlier results are based. ${ }^{1}$

We first checked for the presence of the bulk transition line on hypercubic lattices as large as $L=T=40$ by simulating at $\beta_{\text {adj }}>1.25$. We monitored local observables such as the fundamental and adjoint plaquettes and the Polyakov loops in the four directions, together with their normalised susceptibilities. The bulk transition manifests itself with a jump in the expectation value of the plaquettes as the couplings are varied in the (pseudo)critical region. Moreover, in the same region a clear hysteresis cycle in the plaquettes appears when Markov chains are started from random (hot) or unit (cold) gauge configurations. The presence of metastable states characterised by different values of the plaquette allows us to follow the bulk transition line and estimate the location of its end-point, where the plaquette gap disappears in the thermodynamic limit.

The fundamental plaquette is shown in figure 2 for several $\beta_{\text {fund }}$ values in the pseudocritical range at fixed $\beta_{\text {adj }}=1.275$. A similar behaviour is found for the vacuum expectation value of the modulus of the Polyakov loop. As expected, increasingly larger lattice volumes are requested to correctly identify the presence of the two metastable states (and consequently of the hysteresis cycle) when $\beta_{\text {adj }}$ approches 1.25 from above. However, we noted that the transition to the asymptotic regime is sharp, with values of relevant observables approximately independent of $L$ and $T$ as soon as the small volume regime is exited. On

\footnotetext{
${ }^{1}$ An alternative algorithm with an efficiency similar to the one used in this work has been proposed in [43].
} 


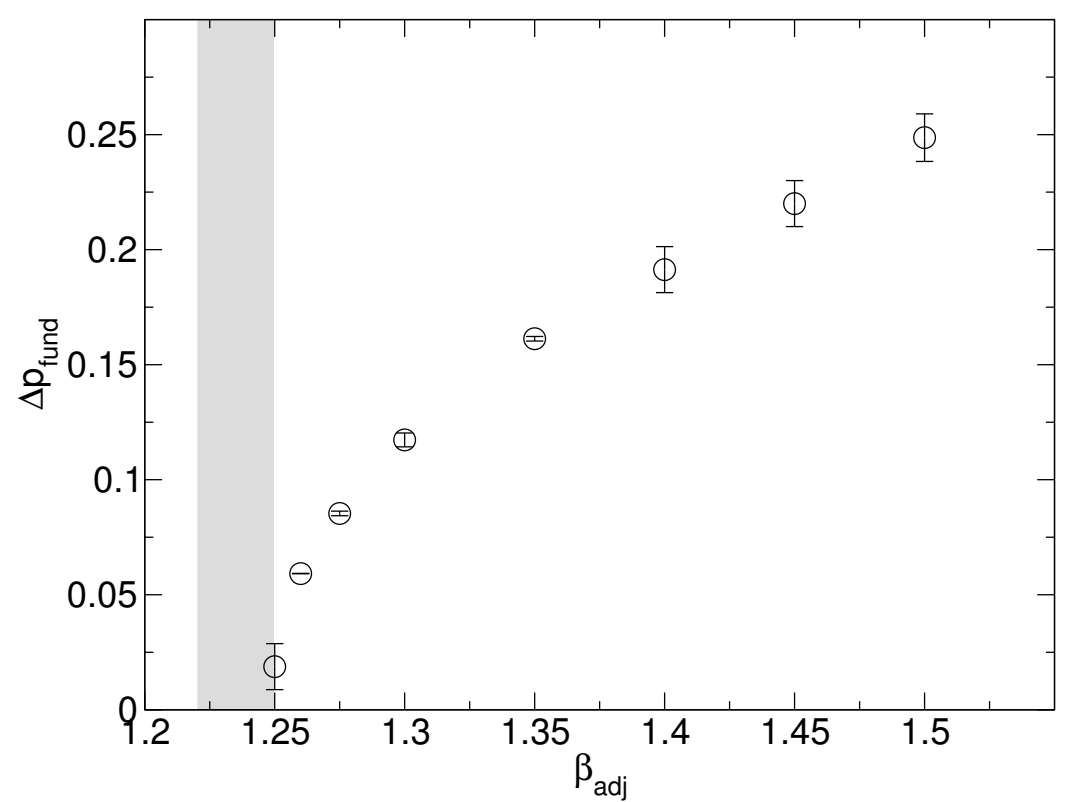

Figure 3. $\Delta p_{\text {fund }}$ as defined in eq. (3.1). Also shown as a grey shaded area is the approximate position of the critical $\beta_{\text {adj }}$ value at which $\Delta p_{\text {fund }}$ is expected to vanish: $1.22<\beta_{\text {adj }}<1.25$. A consistent result is found using the adjoint plaquette $\left(\Delta p_{\text {adj }}\right)$. The plotted points are reported in table 1 .

the largest volume used for this part of the study, for which $L=T=40$, and with more than 400000 measurements, we find that the plaquette gap between the two vacua is nonzero at $\beta_{\text {adj }}=1.25$, in contrast with the results at smaller $L$ of ref. [35]. A careful study of the plaquette gap, defined as

$$
\Delta p_{\text {fund }}=\left\langle\operatorname{Plaq}_{F, 1}\right\rangle-\left\langle\operatorname{Plaq}_{F, 2}\right\rangle
$$

where the subscripts 1 and 2 refer to the distinct vacua at couplings centered in the hysteresis loop, shows a definite trend towards zero. The trend in $\Delta p_{\text {fund }}$ is plotted in figure 3 , where, for each estimate, we used the smallest volume where the first order nature of the transition was manifest. Numerical values are summarised in table 1 . We note that the autocorrelation time dramatically increases as one approaches the critical point (see the $\tau_{f}$ and $\tau_{a}$ columns of table 2). We were unable to give an estimate of the autocorrelation time at $\beta_{\text {adj }}=1.25$, where a large $L=40$ lattice was required. Hence, on this latter point the systematic error is not fully under control. For this reason the point at $\beta_{\text {adj }}=1.25$ will not be considered in our subsequent analyses.

By interpolating both $\Delta p_{\text {fund }}$ and the equivalent quantity $\Delta p_{\text {adj }}$ for the adjoint plaquette using polynomials, we identify a region $1.22<\beta_{\text {adj }}<1.25$ where the plaquette gap vanishes. This is our first estimate for the location of the end-point. This estimate will be refined in the following using scaling analysis of the susceptibilities and of the spectral observables.

In the region below the approximate location of the end-point, we have checked that the transition becomes a crossover, signalled by the lack of volume scaling in the fundamental 
and adjoint plaquette susceptibilities $\chi_{\mathrm{Pf}}, \chi_{\mathrm{Pa}}$ : the height and the location of peaks of the susceptibility (determined with a scan in $\beta_{\text {fund }}$ at fixed $\beta_{\text {adj }}$ ) are consistent across the different volumes. An example is reported in figure 4 . The location of the peak can be followed in the $\left(\beta_{\text {fund }}, \beta_{\text {adj }}\right)$ plane and separates a strong coupling region at small $\beta_{\text {fund }}$ from a region closer to the weak coupling limit $\left(\beta_{\text {fund }} \rightarrow \infty\right)$. We summarise the maximum values for $\chi_{\mathrm{Pf}}$ and $\chi_{\mathrm{Pa}}$ along this crossover line in table 2 . In the same table we also report an estimate of the integrated autocorrelation times for the fundamental and adjoint plaquettes.

The peak of the plaquette susceptibilities can be used to give a new estimate of the end-point location. In a similar analysis, the conventional way of proceeding is to use reweighting to locate the maximum of susceptibilities. We have attempted this procedure, but reweighting proves to be unviable due to the small overlap of the plaquette distributions at neighbour $\beta_{\text {fund }}$ for lattices of the required size. This situation is depicted in figure 5 , where the small overlap is visible for relative variations in $\beta_{\text {fund }}$ that are less than one part in a thousand. Since carrying out a reweighting programme in the critical region will be computationally prohibitive, we reverted to an estimate of the maximum involving a comparison of values at neighbour simulated $\beta_{\text {fund }}$.

Our results show that by approaching $\beta_{\text {adj }} \approx 1.25$ from below, the maximum of the susceptibility increases, at fixed $L^{4}$ volume. Its scaling form can be fitted by

$$
\chi_{\mathrm{Pf}}^{(\max )}=A\left(\beta_{\mathrm{adj}}^{(\mathrm{crit})}-\beta_{\mathrm{adj}}\right)^{-\gamma},
$$

with $A=0.077(5), \beta_{\text {adj }}^{\text {(crit) }}=1.2460(38)$ and $\gamma=1.06(5)\left(\chi^{2} /\right.$ dof $\left.=0.67\right)$ for the data $\beta_{\text {adj }}=$ $1.05-1.22$, at $L=20$. Our numerical data for $\chi_{\mathrm{Pf}}^{(\max )}$ and their best fit are shown in figure 6 . $\chi_{\mathrm{Pf}}^{(\max )}$ is the integrated plaquette-plaquette correlation function. The compatibility within 1.2 standard deviations of the fitted value $\gamma=1.06(5)$ with $\gamma=1$ (predicted by the meanfield theory) gives a first hint that the model could be in the universality class of the $4 \mathrm{~d}$ Gaussian model, whose critical properties are described by the mean-field approximation. ${ }^{2}$ The fitted $\beta_{\text {adj }}^{\text {(crit) }}$ provides another estimate of the end-point location, which is compatible with the value obtained from $\Delta p_{\text {fund }}$. The stability of the fit is checked by changing the number of points included and the fitted parameters are summarised in table 3.

One can also study the scaling as a function of $\beta_{\text {fund }}$. Since our calculations were designed for the scaling in terms of $\beta_{\mathrm{adj}}$, our resolution for this analysis is not optimal, because $\beta_{\text {fund }}$ is measured rather than inputed. This affects most the $\beta_{\text {fund }}$ values far from the critical point, and in particular $\beta_{\text {fund }}=1.40-1.42$. Hence, we use this analysis only as a consistency check of general scaling behaviour. In terms of $\beta_{\text {fund }}$, the scaling form of $\chi_{\mathrm{Pf}}^{(\max )}$ is given by

$$
\chi_{\mathrm{Pf}}^{(\max )}=A\left(\beta_{\text {fund }}-\beta_{\text {fund }}^{(\text {(crit })}\right)^{-\gamma} .
$$

Our best fit (displayed with the numerical data in figure 7) gives $A=0.060(6), \beta_{\text {fund }}^{\text {(crit) }}=$ $1.2229(31)$ and $\gamma=1.03(6)\left(\chi^{2} /\right.$ dof $\left.=1.41\right)$ for the data at $\beta_{\text {fund }}=1.241-1.37$ and $L=20$. Table 4, which reports the values of the fitted parameters for various choices of the fit range,

\footnotetext{
${ }^{2}$ Note that in four dimensions the critical exponents of the Gaussian model are the same as those of the Ising model, and in both models they coincide with the mean-field exponents.
} 


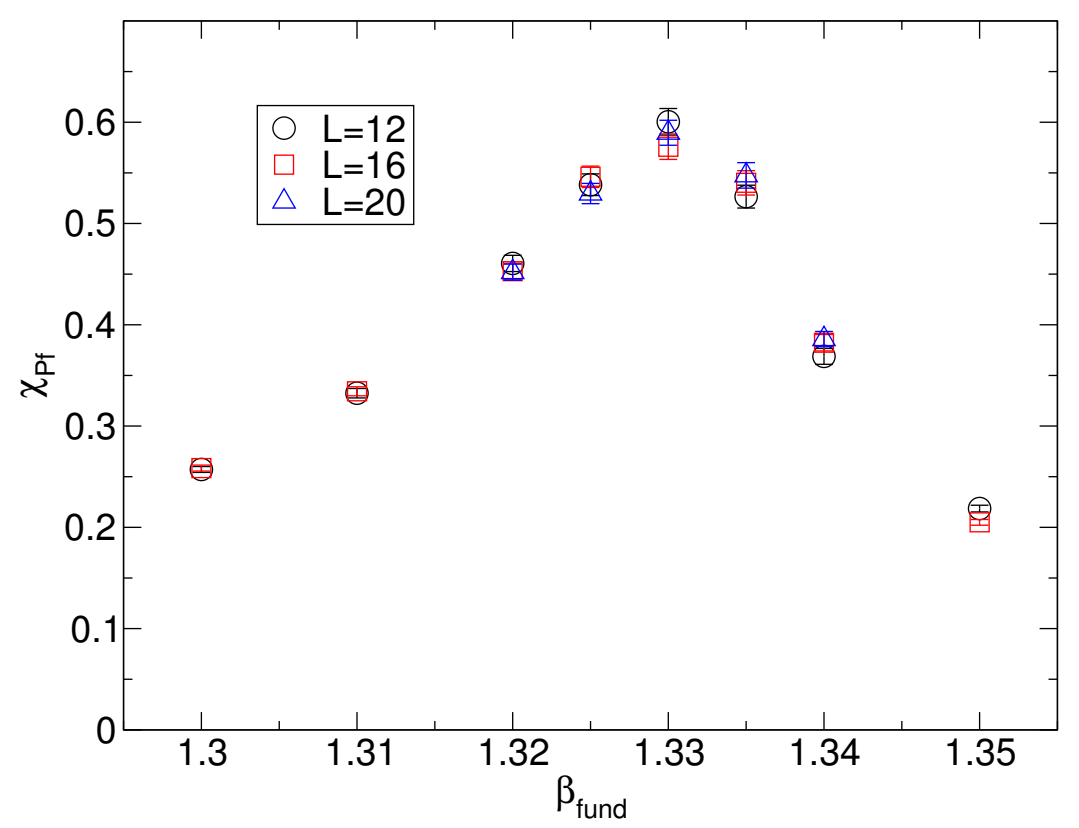

Figure 4. Lack of volume scaling in the fundamental plaquette susceptibility at $\beta_{\mathrm{adj}}=1.10$. The peak location does not move up to volumes $L \times T=20^{4}$.

suggests that the extracted value of the critical coupling and of the critical exponent remain stable across the different fits. Finally, despite using the same notation, we remark that the exponents $\gamma$ in eqs. (3.2) and (3.3) need not to be the same. The fact that the measured values are consistent within errors is a likely indication that both the $\beta_{\text {adj }}$ and the $\beta_{\text {fund }}$ directions have a non-zero projection along the dominant direction of the renormalisation group flow at the relevant infrared fixed point.

\section{Spectrum measurements}

The properties of the spectrum and the scaling of different masses as the critical surface of a fixed point is approached give important information on the low-energy dynamics of the theory. In the following we explain our analysis of the low-lying spectrum in the crossover region. Since this is the first study of this kind, we focused on controlling possible sources of systematic errors such as autocorrelations and finite-size effects. The aim is to extract the light glueball spectrum in the thermodynamic limit and to study the scaling properties of ratios of masses while approaching the end-point in a controlled manner, e.g. along a specified trajectory in the bare parameter space.

We simulate the $\mathrm{SU}(2)$ Yang-Mills theory at six different adjoint couplings $\beta_{\mathrm{adj}}=$ $1.00,1.05,1.10,1.16,1.18,1.20$. For each of them we simulate a range of fundamental couplings $\beta_{\text {fund }}$ such that both the strong coupling and the weak coupling limits are investigated. The location of the simulated points in the two-dimensional space of the couplings is plotted in figure 8 , together with the location of the bulk phase transition and its estimated end-point (cfr. section 3 ). We use several lattice volumes ranging from $6^{3} \times 12$ to $48^{3} \times 48$ in order to try and reach the thermodynamic limit. Thanks to the 


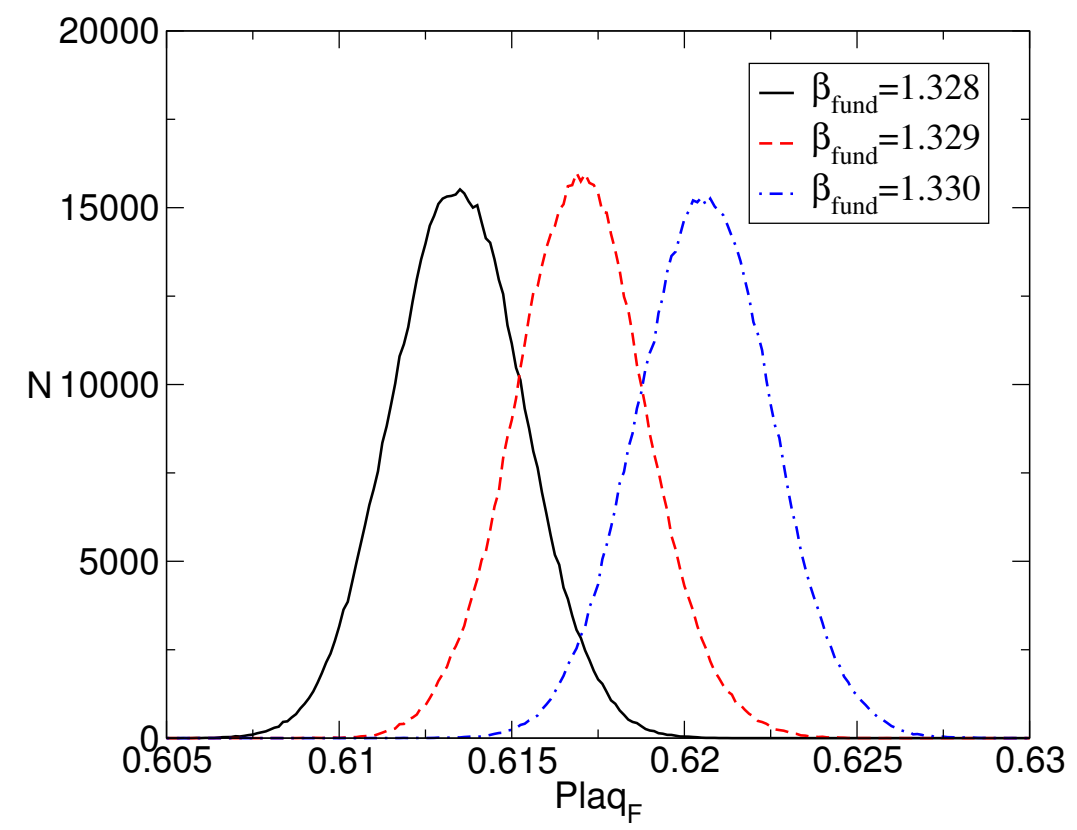

Figure 5. Fundamental plaquette distributions at $\beta_{\mathrm{adj}}=1.10$ for $L \times T=20^{4}$. The overlap of different distributions is not enough to allow for a stable multi-histogram reweighting analysis of the susceptibility. Note that the distance between the points is much finer than the one in figure 4 .

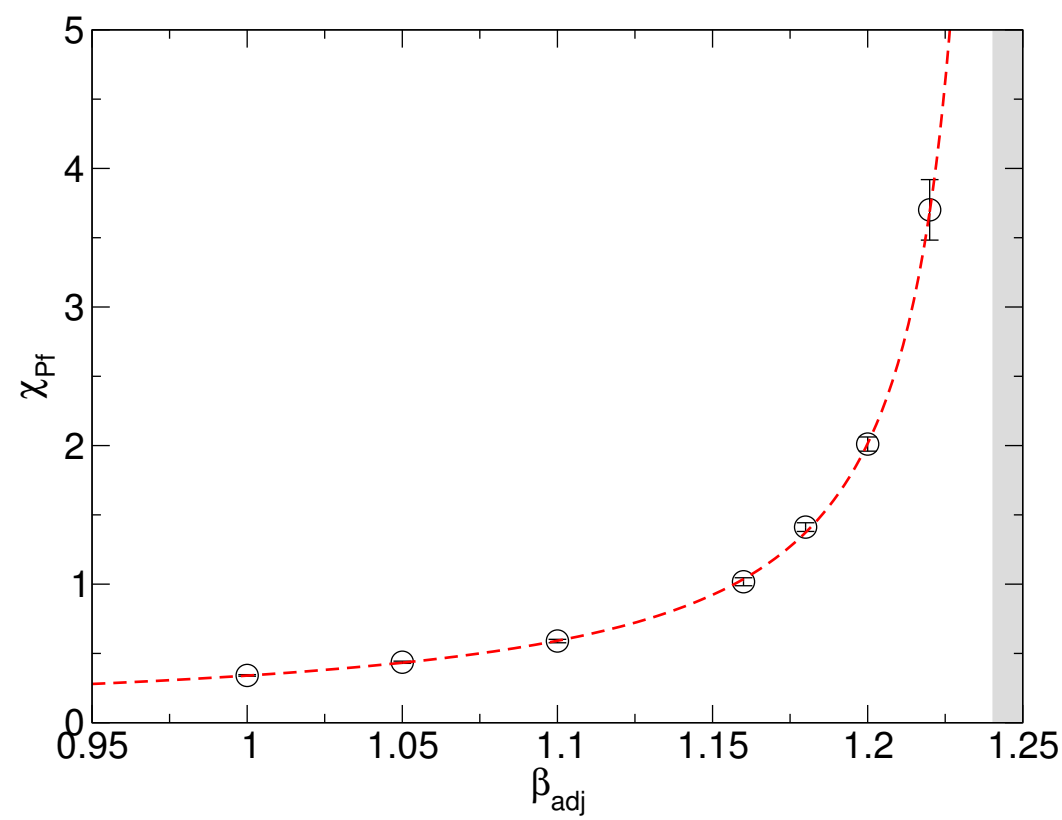

Figure 6. Scaling of the fundamental plaquette susceptibility maximum values for different $\beta_{\text {adj }}$ couplings toward the location of the bulk transition end-point. The estimated $\beta_{\text {adj }}^{(\text {crit }}$ from a fit with eq. (3.2) is highlighted by the grey shaded region on the right.

study of the local observables needed for the phase diagram, we were able to estimate the autocorrelation times and we noted a drastic increase of them for $1.16 \leq \beta_{\text {adj }} \leq 1.20$ (cfr. table 2). Therefore, different measurements are separated by $N_{\tau}$ gauge updates to reduce 


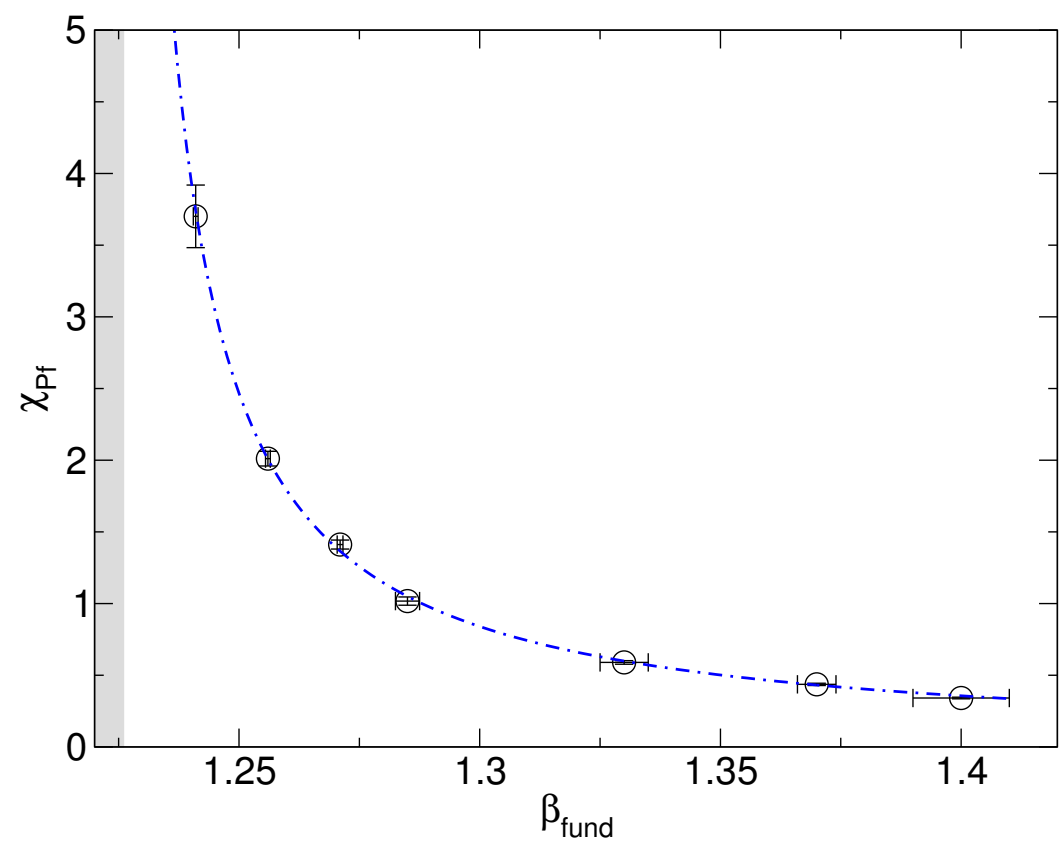

Figure 7. Scaling of the fundamental plaquette susceptibility maximum values for different $\beta_{\text {fund }}$ couplings toward the location of the bulk transition end-point. The estimated $\beta_{\text {fund }}^{\text {(crit) }}$ from a fit with eq. (3.3) is highlighted by the grey shaded region on the left.

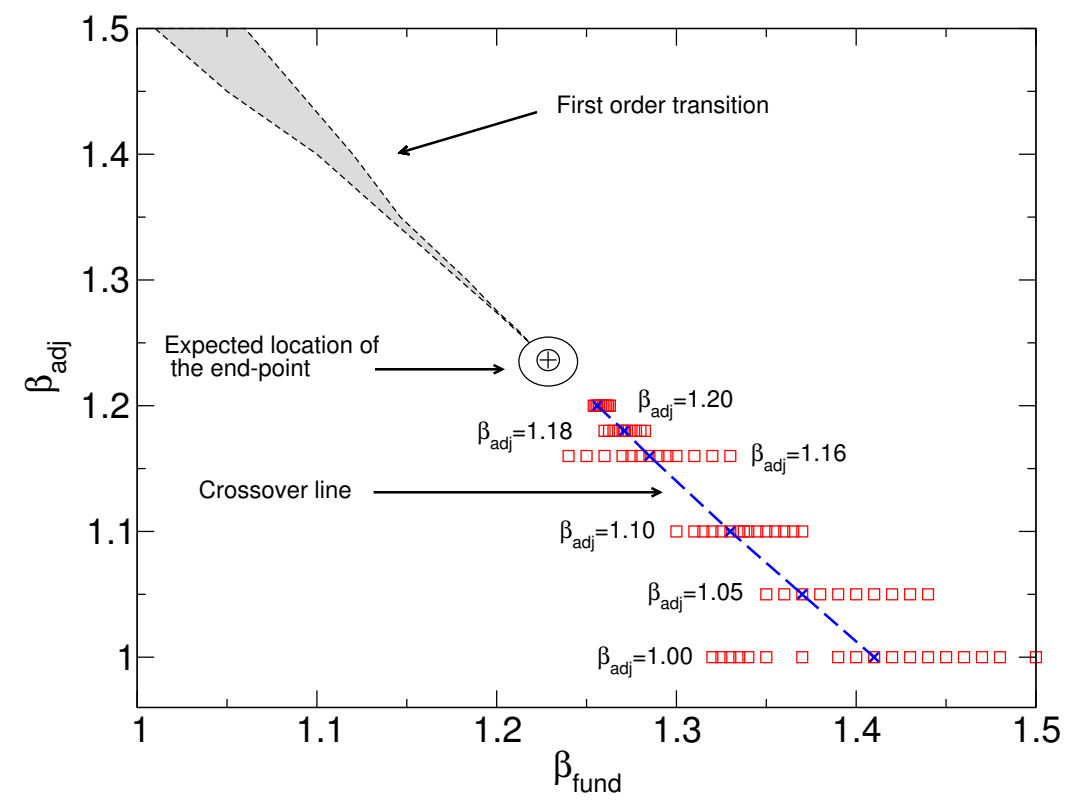

Figure 8. Location of the bulk phase transition (grey area delimited by thin dashed black lines) and of the points where we measured the spectrum of the theory (red squares). The estimated location of the bulk transition end-point is indicated by concentric circles. The thick dashed blue line joins the points where $\chi_{\mathrm{Pf}}$ reaches its maximum and defines a crossover region.

autocorrelations, with $N_{\tau}=250$ at $\beta_{\text {adj }}=1.16$ up to $N_{\tau}=800$ at $\beta_{\text {adj }}=1.20$. Large statistics ensembles with $N_{\text {meas }} \geq 10000$ measurements allow us to extract masses of gluon bound states with great accuracy. A variational ansatz using a large basis of interpolating 


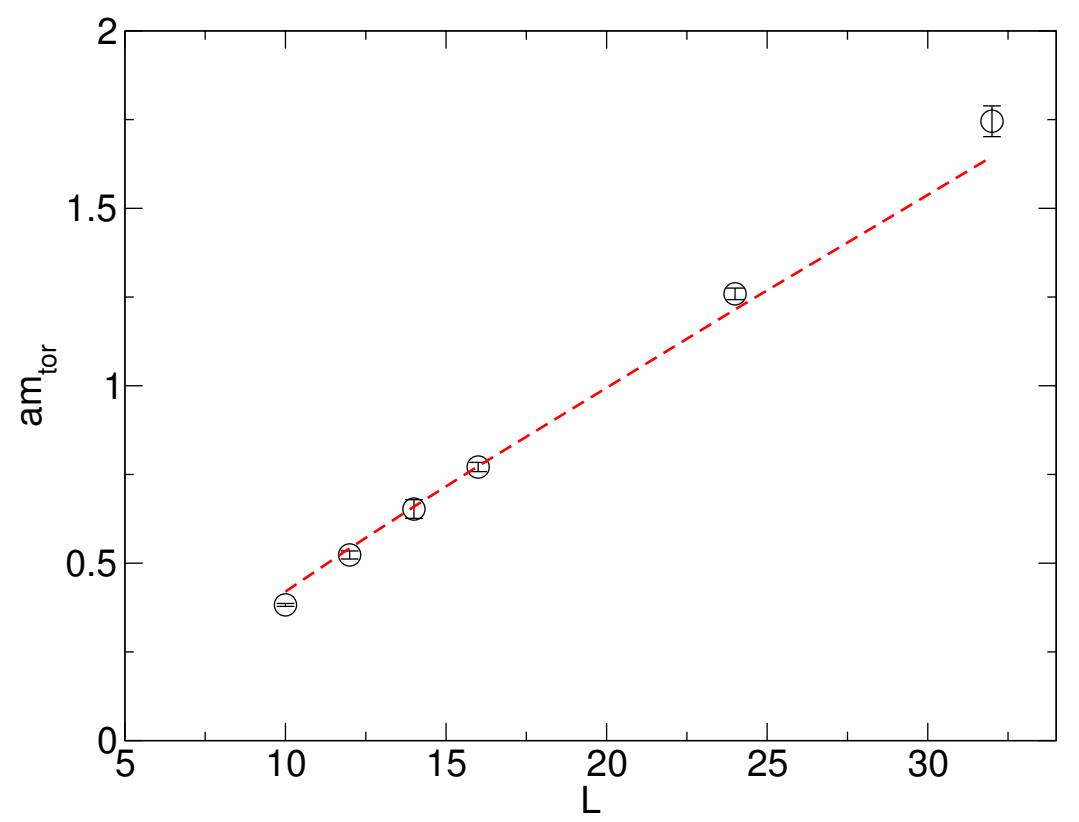

Figure 9. Dependence of the torelon mass $a m_{\text {tor }}$ on its length $L$ at fixed $\beta_{\text {adj }}=1.20, \beta_{\text {fund }}=1.259$. The dashed line is eq. (4.1) with $a \sqrt{\sigma}=0.2290$, obtained from $a m_{\text {tor }}(L)$ at $L=16$. Torelon masses larger than the cutoff are not considered as reliable estimates.

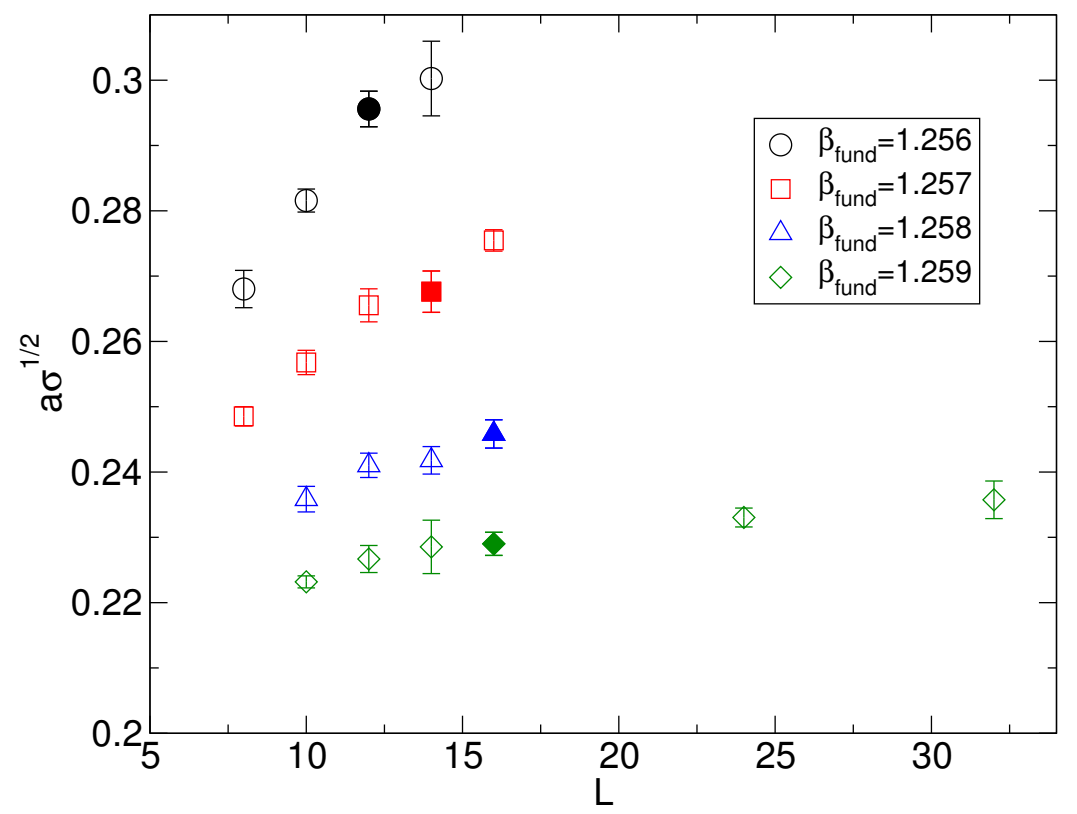

Figure 10. Dependence of the string tension $a \sqrt{\sigma}$ on the length of the spatial torelons $L$ at fixed $\beta_{\text {adj }}=1.20$ and for different fundamental couplings. The filled points identify the results used to estimate the infinite volume limit of $a \sqrt{\sigma}$.

operators is employed to extract the ground state and excited state masses in different symmetry channels as described in ref. [44].

First of all, we extracted the string tension $a \sqrt{\sigma}$, which was then used to set the overall scale. This measure of the dynamical mass gap is extracted from long spatial Polyakov 


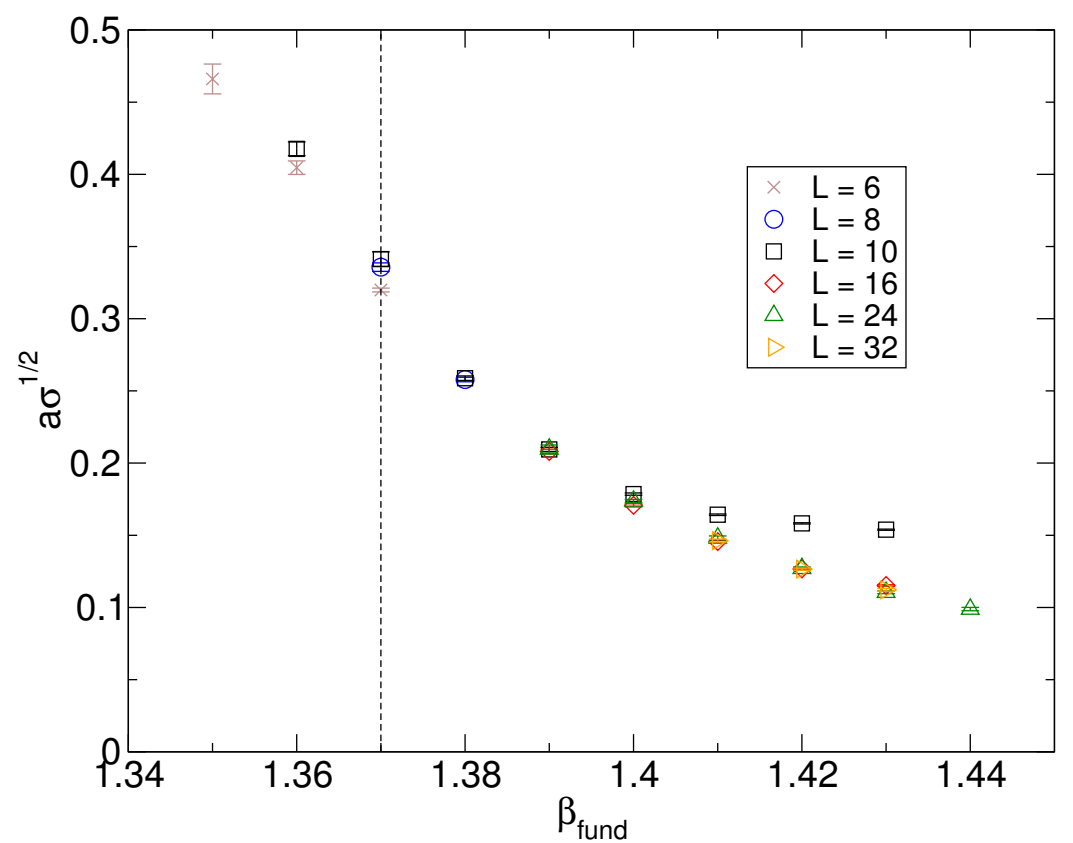

Figure 11. String tension measured from several different volumes $L=6-32$ at $\beta_{\text {adj }}=1.05$. At weaker coupling $\beta_{\text {fund }}>1.39$ larger volumes are needed to keep finite-volume effects under control. The dashed vertical line indicates the approximate position of the maximum in $\chi_{\mathrm{Pf}}$.

loop correlators. The asymptotic large-time behaviour of these correlators is governed by the lightest torelon state. Assuming that a confining flux tube with massless modes binds a static quark-antiquark pair, the mass of the lightest torelon $a m_{\text {tor }}$ can be used to obtain the string tension according to the ansatz

$$
\operatorname{am}_{\text {tor }}(L)=a^{2} \sigma L-\frac{\pi}{3 L}-\frac{\pi^{2}}{18 L^{3}} \frac{1}{a^{2} \sigma} .
$$

The validity of the above equation is checked a posteriori by comparing the extracted string tension at various sizes $L$ and by evaluating the size of the subleading finite $L$ correction $c_{2}=\pi^{2} /\left(18 L^{3} a^{2} \sigma\right)$ with respect to the leading one $c_{1}=-\pi /(3 L)$. This procedure is illustrated on a typical set of data in figure 9, where the string tension is not fitted, but rather extracted using eq. (4.1) and the data point $a m_{\text {tor }}(L)$ at $L=16$. We observe that, when the loop is too short (i.e. such that $a m_{\text {tor }}(L)<0.5$ ), the asymptotic formula relating string tension, length of the loop and torelon mass fails to describe the latter. On the other hand, extracting masses that are above the lattice cutoff is subject to systematic errors. There is an intermediate region of masses, $0.5 \leq a m_{\text {tor }}(L) \leq 1.0$, that are correctly described by eq. (4.1). For the numerical value at the highest simulated $L$ in the regime of validity of eq. (4.1) (which in this case is the result at $L=16$ ) one gets $c_{2} / c_{1} \simeq 0.039$. Besides justifying our procedure, these results give support to the existence of confining fluxes below the critical point (see also figure 10, showing an example of the variation of $a \sqrt{\sigma}$ as a function of $L$ and $\beta_{\text {fund }}$ at fixed $\beta_{\text {adj }}$ ). Our results show that significant finitesize effects are absent when $L a \sqrt{\sigma}>3$, which we satisfied in our simulations using large spatial volumes for the smallest values of $a \sqrt{\sigma}$. Indeed, in the explored range of couplings, 


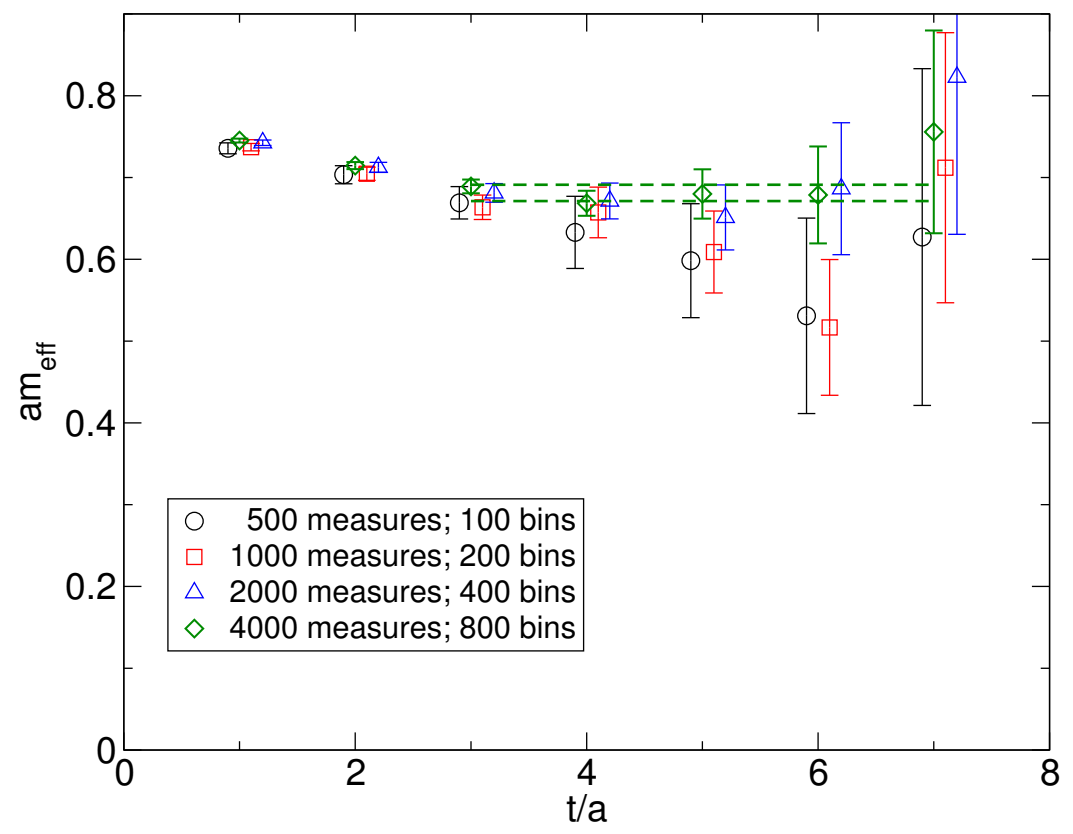

Figure 12. Effective $a m_{\text {tor }}$ at $\beta_{\text {adj }}=1.20$ and $\beta_{\text {fund }}=1.256$ for $L=10, T=20$. The statistics is doubled until a plateaux is clearly identified. The dashed green lines indicate the 1- $\sigma$ contour of the fitted effective mass between $t=3$ and $t=7$. Data points are horizontally shifted for clarity.

the string tension can change by a factor of 5 and, whereas small $L \sim 8-10$ volumes are sufficient at stronger couplings, larger ones are needed towards weak coupling. The situation is shown in figure 11 and it is representative of all the simulated $\beta_{\text {adj }}$ points.

We can estimate the infinite-volume limit of this observable in the following way: when the two largest simulated volumes at each point give consistent results within two standard deviations, we take the largest volume result as an estimate of the thermodynamic limit (provided $a m_{\text {tor }}$ is below the cutoff). When the aforementioned criterion is not fulfilled, we do not give an infinite-volume estimate. However, if a single volume simulation is available, we still report it in plots that show results on various volumes. We use the same approach for estimating the large volume limit also for the other spectral observables studied in this section.

Another important point that we mentioned in section 3 is the increase in the number of measurements that are needed closer to $\beta_{\text {adj }} \approx 1.25$ due to large autocorrelation times. For example, in figure 12 we show that almost a tenfold increase in statistics is needed to reduce the systematic error in the identification of the effective mass plateaux for $a m_{\text {tor }}$ at

Our second spectral observable is the mass of the scalar glueball state $a m_{0^{++}}$. In order to measure this mass, the vacuum subtracted correlators of smeared Wilson loops, symmetrised to have $0^{++}$quantum numbers, have been inserted in a variational basis for a generalised eigenvalue problem. In addition, in order to identify finite-size artefacts, a different type of scalar operators made by symmetrised Polyakov loops winding in opposite directions around the spatial torus has been used in the same variational calculation. This second operator set couples mainly to bi-torelon excitations, which are suppressed in 


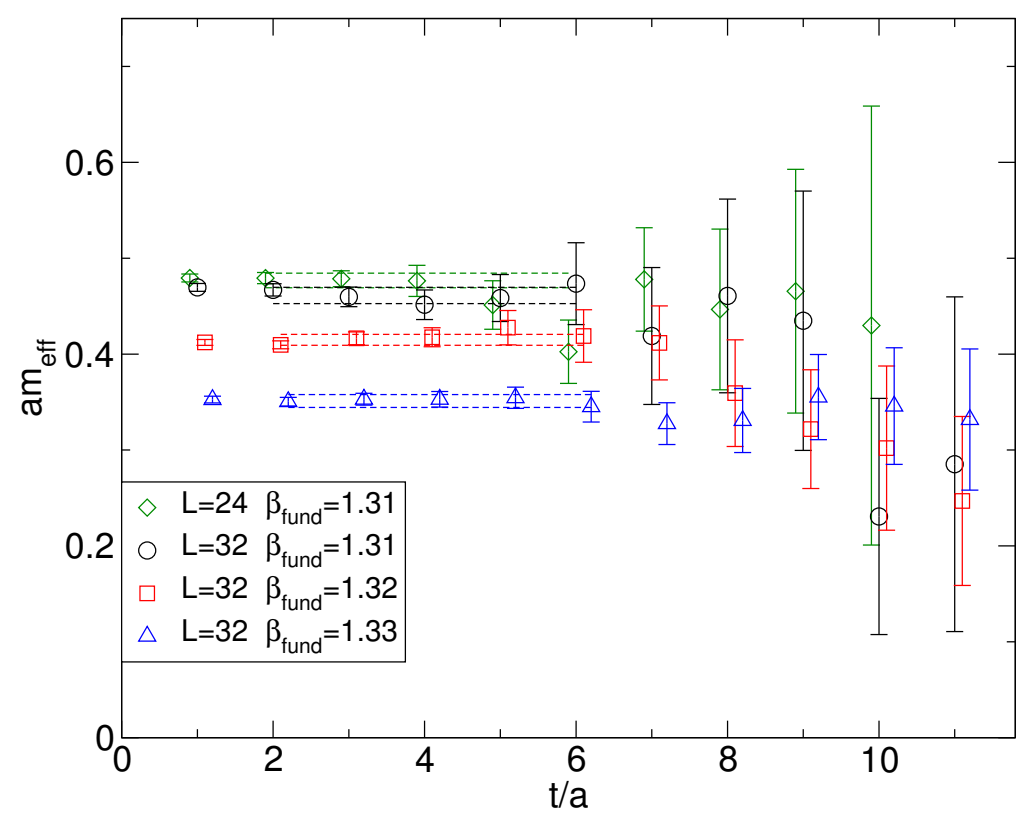

Figure 13. Scalar glueball effective mass at $\beta_{\text {adj }}=1.16$ and for three values of $\beta_{\text {fund }}$. A comparison between $L=24$ and $L=32$ results for $\beta_{\text {fund }}=1.31$ is also shown. The $1-\sigma$ contour of the fitted masses is plotted on top of the respective fitted points. Data points are horizontally shifted for clarity.

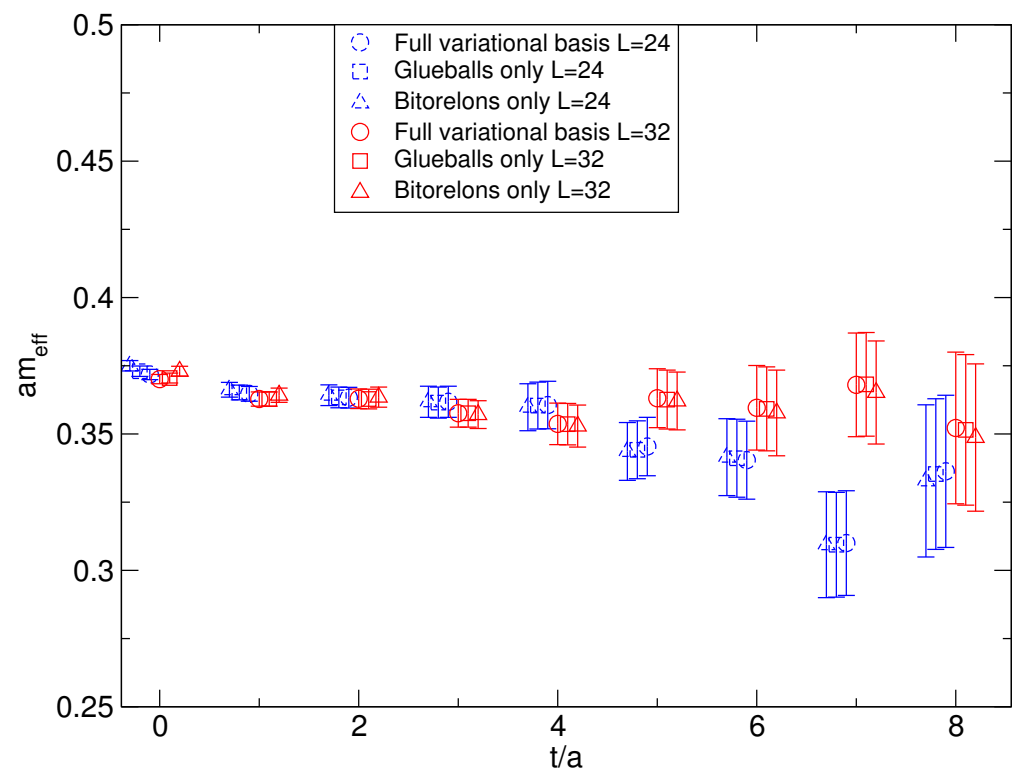

Figure 14. Effective mass of the scalar ground state obtained using different variational basis. Two volumes are compared at $\beta_{\text {fund }}=1.259, \beta_{\text {adj }}=1.20$. No significant difference is present. Points are shifted for clarity.

the large volume limit and can be used to identify these spurious contaminations of the spectrum in the scalar channel. For further technical details, we refer the reader to ref. [44].

The scalar glueball mass is reliably estimated thanks to the large variational operator basis used in our calculation, which allows us to obtain long and robust effective mass 


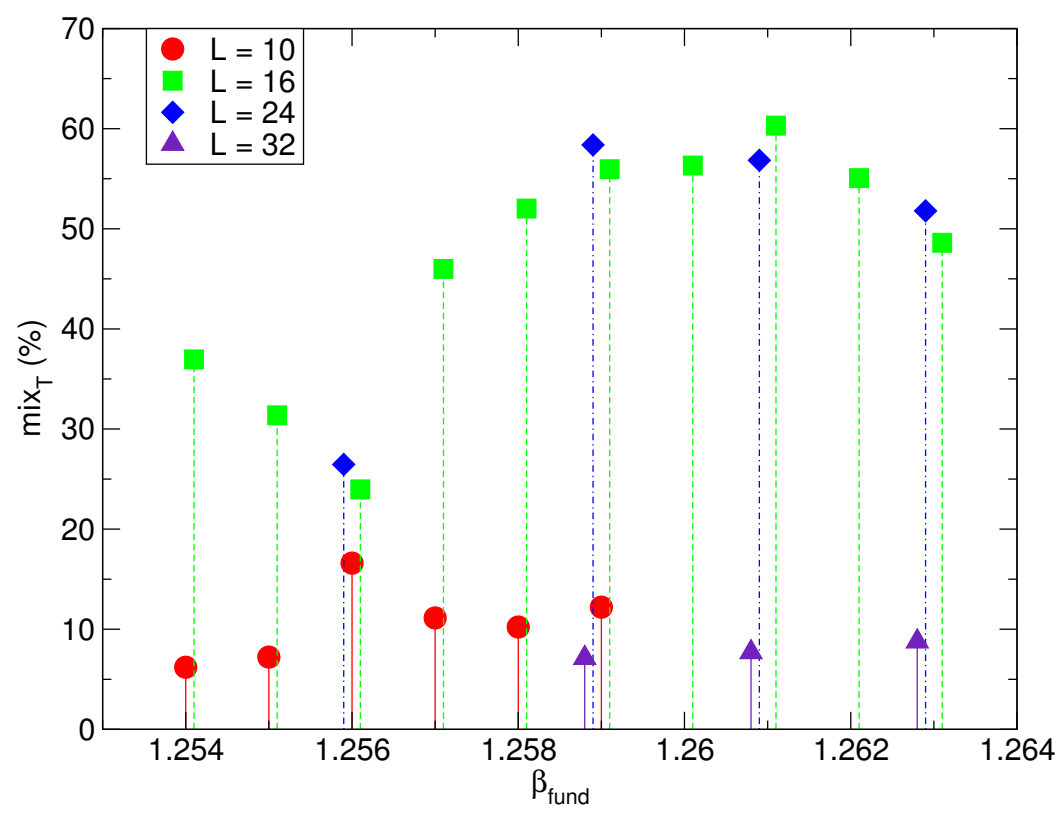

Figure 15. Relative contribution of the bi-torelon operators to the scalar ground state for several fundamental couplings at $\beta_{\mathrm{adj}}=1.20$. On the largest volume, this contribution drops significantly. Values at different volumes are shifted horizontally for clarity.

plateaux. Figure 13 provides an example of effective masses for a large $32^{4}$ lattice at fixed $\beta_{\text {adj }}$ for three $\beta_{\text {fund }}$ values, with a comparison with results from a smaller $24^{3} \times 32$ for one value of $\beta_{\text {fund. }}$. For larger $\beta_{\text {fund }}$ values, we used bigger volumes in order to check explicitly for finite-size effects. Moreover, the contributions of spurious states has been investigated by looking at the extracted spectrum using the different kind of operators described above. Surprisingly, for some values of the couplings we have noticed a large $\mathcal{O}(50 \%)$ contribution of the bi-torelon operators to the ground state; a variational analysis containing only Wilson loop operators or, separately, only Polyakov loop operators, turned out to give the same results for the ground state masses. An example of the effective mass plot obtained from such different variational operator bases is shown in figure 14. This confirms that bi-torelon operators can give sizeable contributions to correlators used to extract the scalar ground state mass. However, by using larger lattices we could confirm that the contribution of these operators dropped down to less than $10 \%$, as expected. This is clearly depicted in figure 15 , where the relative bi-torelon operators contribution to the ground state is shown at $\beta_{\mathrm{adj}}=1.20$ and for several volumes. In our computation, care has been taken in choosing the lattice size in such a way that bi-torelon contamination in the scalar spectrum is negligible. Results for the infinite volume limit of $a \sqrt{\sigma}$ and $a m_{0^{++}}$ at various fundamental and adjoint couplings are included in table 5 to 10 .

The strategy applied for the extraction of the tensor glueball mass $a m_{2^{++}}$is similar to that used for $a m_{0^{++}}$. Here, the lattice operators are symmetrised to project only onto the $E$ irreducible representation of the cubic group which is subduced from the spin 2 of the full continuum rotational symmetry. We remark that for some of the $\beta_{\text {adj }}$ couplings we could 


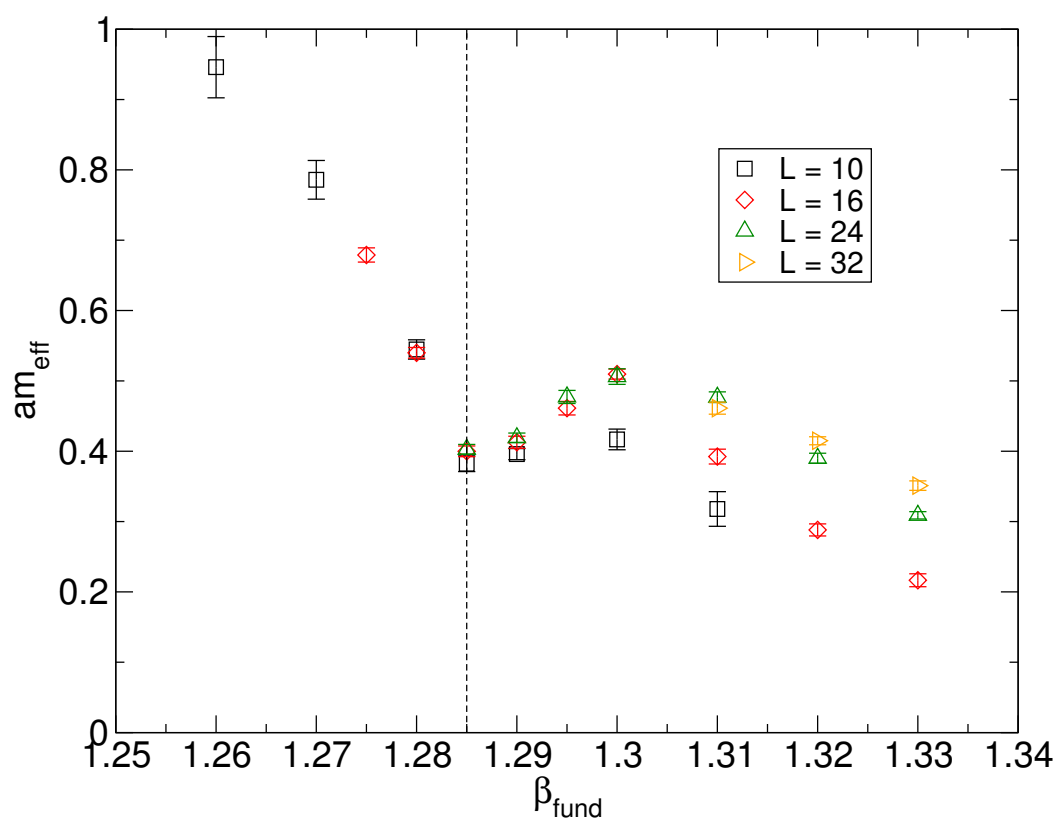

Figure 16. Scalar glueball mass at $\beta_{\mathrm{adj}}=1.16$ for a wide range of fundamental couplings. Contrary to the monotonicity of $a \sqrt{\sigma}$ in the same range of $\beta_{\text {fund }}, a m_{0^{++}}$develops a dip in the crossover region, before raising and decreasing again in the weak coupling limit. The dashed vertical line indicates the approximate position of the maximum in $\chi_{\mathrm{Pf}}$.

not reliably estimate the thermodynamic limit of $a m_{2^{++}}$due to somewhat larger finite-size effects. It is known that this channel is more difficult to extract due to the heavier mass of its ground state. Results in the thermodynamic limit are summarised in table 11 for this observable.

\section{$5 \quad$ Scaling properties}

Let us move to the description of the features of the extracted spectrum, when the relavant sources of finite-size effects are taken into account. The most interesting feature of the scalar channel spectrum is the presence of a slight dip for a certain region of $\beta_{\text {fund }}$ couplings around the crossover region, where $\chi_{\mathrm{Pf}}$ reaches its maximum value. This dip becomes more pronounced and at its bottom the mass value gets lighter as we increase $\beta_{\text {adj }}$ towards the transition end-point. At $\beta_{\text {adj }}=1.00$ the dip is still only a mild plateaux that $a m_{0^{++}}$reaches before starting decreasing again towards the weak coupling region. However, at $\beta_{\text {adj }}=1.16$ $a m_{0^{++}}$drops dramatically, to form the dip shown in figure 16. A similar situation has been found at $\beta_{\text {adj }}=1.18$ and 1.20. It is important to recall that such a behaviour is absent in both $a \sqrt{\sigma}$ and $a m_{2^{++}}$, which smoothly decrease as functions of $\beta_{\text {fund }}$. A situation where the infinite-volume limit has been estimated is shown in figure 17 .

In a neighbourhood of a second order phase transition point the light lattice degrees of freedom that become massless at the critical point define an effective long-distance continuum theory. As the critical point is approached, their mass goes to zero according to some scaling exponents that characterise the dynamics at large distances. For our 


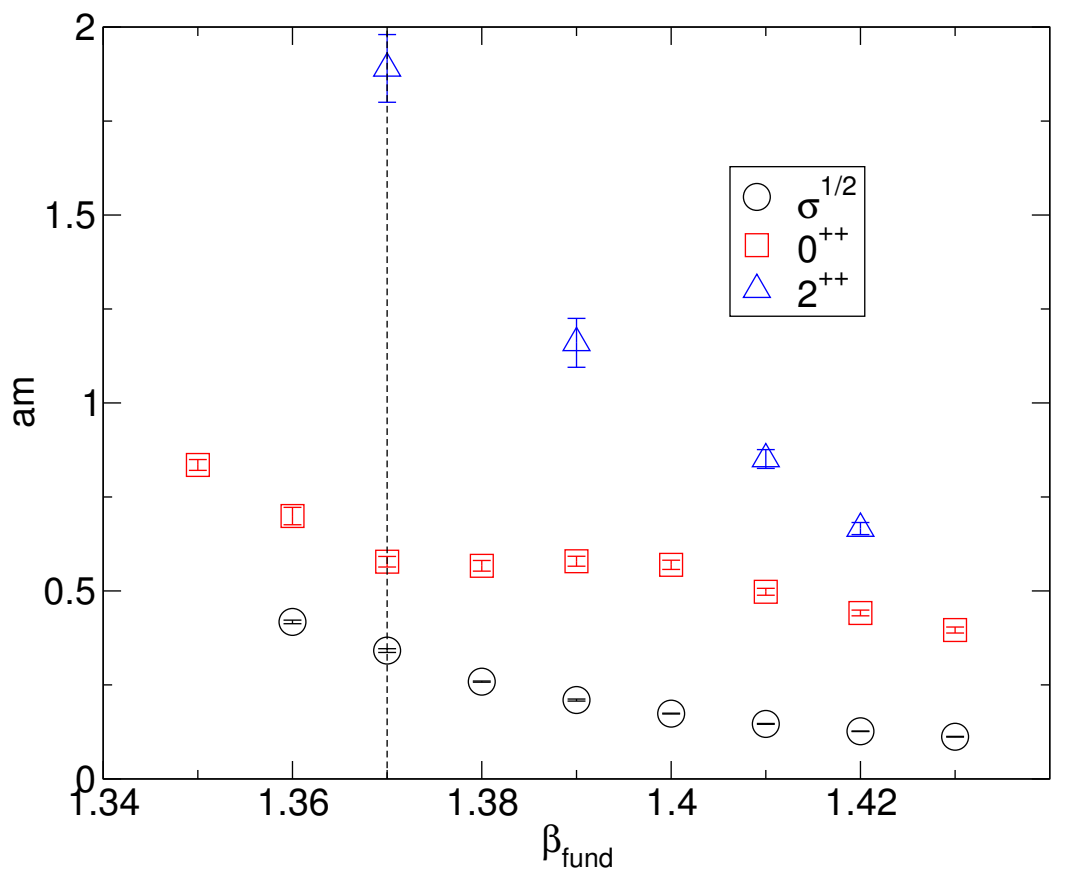

Figure 17. The measured low-lying spectrum of $a \sqrt{\sigma}, a m_{0^{++}}$and $a m_{2^{++}}$at $\beta_{\text {adj }}=1.05$ in the infinite-volume limit. The dashed vertical line indicates the approximate position of the maximum in $\chi_{\mathrm{Pf}}$.

system, only $a m_{0^{++}}$seems to become light at the end-point of the first order line. In order to investigate its approach to the end-point, we fit the measured $a m_{0^{++}}$using the parameterisation

$$
a m_{0^{++}}=A\left(\beta_{\text {adj }}^{(\text {crit })}-\beta_{\text {adj }}\right)^{P},
$$

which is inspired by the scaling of the correlation length $\xi$ near a critical point: $\xi=$ $\xi_{0}\left|T / T_{c}-1\right|^{-\nu}$. The critical exponent $\nu$ is 0.5 for the $4 \mathrm{~d}$ Gaussian model (mean-field theory). By fitting the three free parameters using all the available data, we obtain $A=$ $1.19(5), \beta_{\text {adj }}^{\text {(crit) }}=1.2308(59)$ and $P=0.42(3)$ with a good $\chi^{2} /$ dof $=1.07$. We also fitted the data keeping a fixed $P=0.5$ to test the mean-field hypothesis and we obtain $A=1.31(5)$, $\beta_{\text {adj }}^{\text {(crit) }}=1.2455(26)$ with a worsened $\chi^{2} /$ dof $=1.97$. Both fits are compared to the data in figure 18. Taken at face value, our results suggest that the mean-field scaling is not ruled out. Indeed if we exclude the point at $\beta_{\text {adj }}=1.00$, the value of $P$ gets closer to the meanfield prediction (table 12). As shown in table 13, a similar analysis of the scaling of $a m_{0^{++}}$ in terms of $\beta_{\text {fund }}$ gives compatible results. To resolve the issue of whether the system is described by the mean-field theory, one would need to go closer to the end-point, which is currently computationally prohibitive for the resources at our disposal. The $m_{0^{++}} / \sqrt{\sigma}$ ratio is shown in figure 19 .

\section{Conclusions}

Motivated by the need to better understand possible roles of lattice artefacts in investigations of gauge theories in the (near-)conformal regime, we have studied a $\mathrm{SU}(2)$ pure 


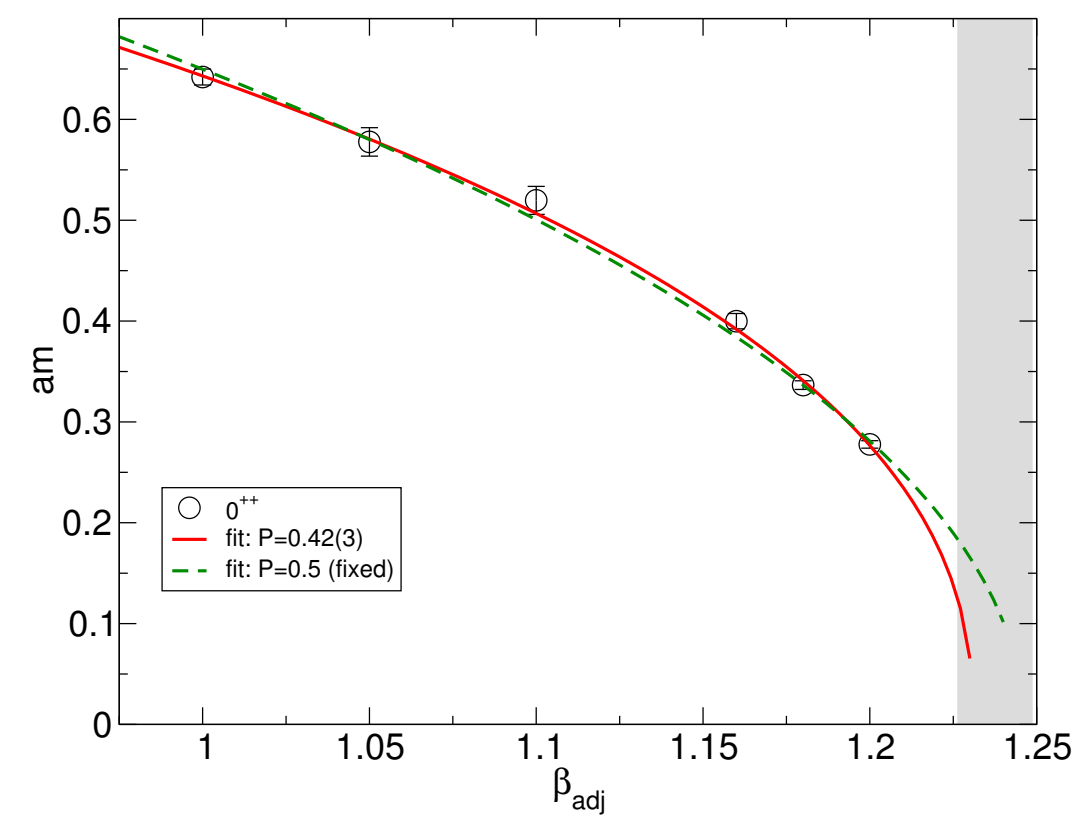

Figure 18. $a m_{0^{++}}$for different values of $\beta_{\text {adj }}$ and on the trajectory defined by the maxima of $\chi_{\mathrm{Pf}}$. The fitting function from eq. (5.1) is used to represent the data. The shaded grey area comprises the values of the critical point coming from the two different fits in the plot.

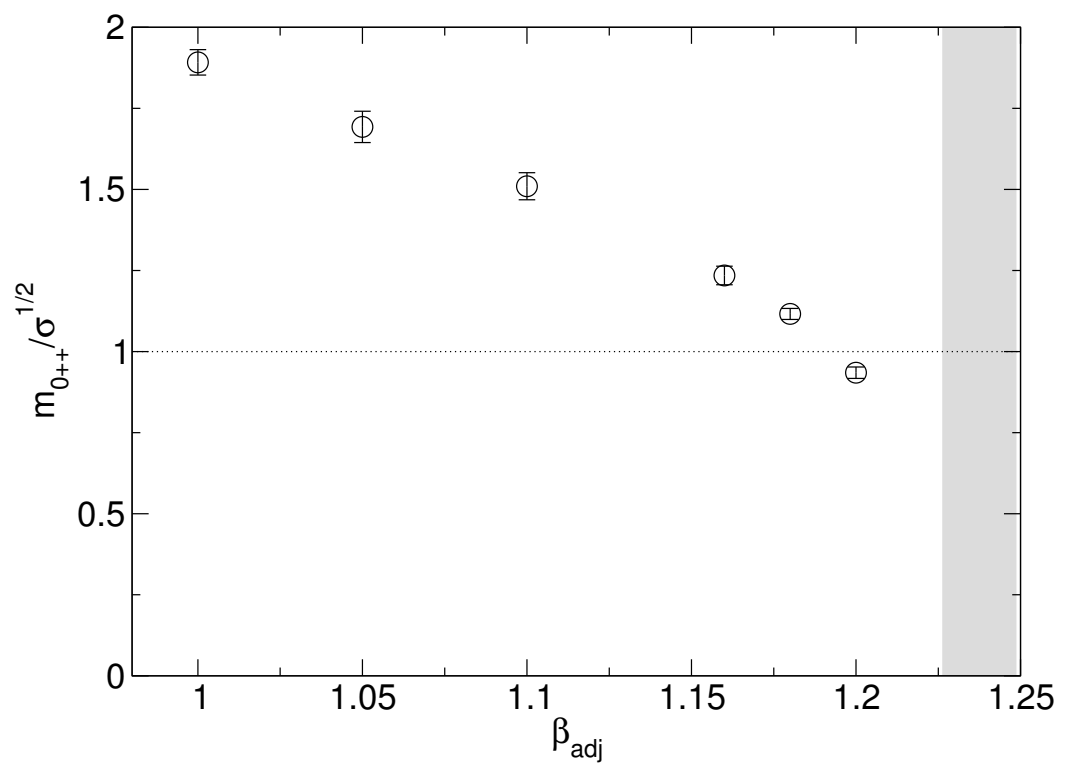

Figure 19. The ratio between $a m_{0^{++}}$and $a \sqrt{\sigma}$ for different values of $\beta_{\text {adj }}$ and on the trajectory defined by the maxima of $\chi_{\mathrm{Pf}}$. The ratio decreases below one when the bulk transition end-point is approached $\left(\Delta p_{\text {fund }}=0\right)$. The shaded grey area indicates the estimated location of the critical point obtained by the scaling analysis (eq. (5.1)).

gauge theory with a modified lattice action with couplings to both the fundamental and the adjoint plaquettes. This theory, which is related to $\mathrm{SU}(2)$ gauge theory with two Dirac flavours in the adjoint representation in the limit of large bare fermion mass, is known to 
have a bulk phase transition with an end-point relatively close to the fundamental coupling axis. The controversial nature of this end-point is resolved and our estimates for its location are summarised in table 14. Using our improved gluon spectroscopy techniques [44], we measured the string tension, the scalar glueball mass and the tensor glueball mass. We studied their scaling properties when the end-point is approached along a controlled trajectory that follows the peaks of the fundamental plaquette susceptibility. To our knowledge, this is the first systematic study of the gluonic spectrum in this model. For this reason, we carefully checked that we are reasonably free from finite-size effects and (mostly thanks to the simulation algorithm used in this work) the autocorrelation time of our observables was kept under control. The spectrum extrapolated to infinite volume shows a non-constant $m_{0^{++}} / \sqrt{\sigma}$ ratio when approaching the end-point in a controlled manner (see figure 19). In particular, since the $0^{++}$state is the only light degree of freedom near the end-point and the scaling is marginally compatible with being described by the critical exponents of the 4d Gaussian model, it is conceivable that the corresponding effective theory be a scalar theory described by the mean-field approximation. This behaviour is in contrast with the infrared dynamics of $\mathrm{SU}(2)$ gauge theory with two adjoint Dirac fermions, where such a ratio is driven to a constant by a conformal fixed point and is consistent with the continuum SU(2) Yang-Mills value $m_{0^{++}} / \sqrt{\sigma} \sim 3.7$. Therefore, we can reasonably conclude that the observed spectral signals of near-conformality in $\mathrm{SU}(2)$ gauge theory with two adjoint Dirac fermions are not affected by the second order phase transition point of the related gauge system with mixed fundamental-adjoint action. It would be instructive to perform a similar analysis for gauge theories with fermions in the symmetric or antisymmetric representation and $N_{c} \geq 4$, for which the stability of fluxes of higher $\mathcal{N}$-lity in pure gauge (see e.g. [45]) could create a more complicated phase structure in the effective theory at large mass.

\section{Acknowledgments}

We would like to thank Philippe de Forcrand, Claudio Bonati and Guido Cossu for fruitful comments and discussions. We are indebted with Urs Heller for guidance on the algorithm used in our simulations. B.L. acknowledges financial support from the Royal Society (grant UF09003) and STFC (grant ST/G000506/1) and the hospitality of the Aspen Center for Physics during the final stage of this work, which allowed him to discuss various aspects of the project with the participants to the workshop Lattice Gauge Theory in the LHC Era, and in particular with R. Brower, A. Hasenfratz, Y. Meurice and T. Tomboulis. E.R. was funded by a SUPA Prize Studentship and a FY2012 JSPS Postdoctoral Fellowship for Foreign Researchers (short-term). The simulations discussed in this work have been performed on a cluster partially funded by STFC and by the Royal Society, on systems made available to us by HPC Wales and on the HPCC Plymouth cluster facilities at Plymouth University.

Open Access. This article is distributed under the terms of the Creative Commons Attribution License which permits any use, distribution and reproduction in any medium, provided the original author(s) and source are credited. 


\begin{tabular}{|c|c|c|c|}
\hline$L$ & $\beta_{\text {adj }}$ & $\beta_{\text {fund }}$ & $\Delta p_{\text {fund }}$ \\
\hline 8 & 1.50 & 1.04 & $0.25(1)$ \\
8 & 1.45 & 1.075 & $0.22(1)$ \\
8 & 1.40 & 1.110 & $0.19(1)$ \\
8 & 1.35 & 1.144 & $0.161(1)$ \\
12 & 1.30 & 1.1815 & $0.117(3)$ \\
16 & 1.275 & 1.1999 & $0.085(1)$ \\
20 & 1.26 & 1.2109 & $0.0592(1)$ \\
40 & 1.25 & $1.2183^{*}$ & $0.02(1)^{*}$ \\
\hline
\end{tabular}

Table 1. The estimated location of the hysteresis centre at different $\beta_{\text {adj }}$ values. Each value $\Delta p_{\text {fund }}$ is measured on a volume $L^{4}$, which is the minimum one needed to discern the two metastable states of the first order transition. The errors on $\Delta p_{\text {fund }}$ are estimated by comparing its value on all the simulated points in the hysteresis region. However, the starred point comes from a single simulation, and the error comes from the difficulty of estimating the expectation values $\left\langle\mathrm{Plaq}_{F, 1}\right\rangle$ and $\left\langle\mathrm{Plaq}_{F, 2}\right\rangle$ in the presence of long autocorrelation times.

\begin{tabular}{|c|c|c|c|c|c|}
\hline$\beta_{\text {adj }}$ & $\beta_{\text {fund }}$ & $\chi_{\mathrm{Pf}}^{(\max )}$ & $\tau_{\mathrm{f}}$ & $\chi_{\mathrm{Pa}}^{(\max )}$ & $\tau_{\mathrm{a}}$ \\
\hline 1.00 & $1.40(1)$ & $0.3415(55)$ & $45(2)$ & $0.2102(30)$ & $40(2)$ \\
$* 1.00$ & $1.41(1)$ & $0.3307(58)$ & $52(3)$ & $0.2261(37)$ & $47(5)$ \\
1.05 & $1.370(4)$ & $0.4366(82)$ & $72(4)$ & $0.2834(49)$ & $66(4)$ \\
1.10 & $1.330(5)$ & $0.590(12)$ & $86(6)$ & $0.3658(77)$ & $81(5)$ \\
1.16 & $1.2850(25)$ & $1.017(29)$ & $175(17)$ & $0.591(17)$ & $168(16)$ \\
1.18 & $1.2710(6)$ & $1.412(32)$ & $276(34)$ & $0.835(18)$ & $244(16)$ \\
1.20 & $1.2560(5)$ & $2.011(51)$ & $401(59)$ & $1.175(29)$ & $366(30)$ \\
1.22 & $1.2410(5)$ & $3.70(22)$ & $623(113)$ & $2.14(13)$ & $617(112)$ \\
\hline
\end{tabular}

Table 2. The maximum of the fundamental and adjoint plaquette susceptibilities $\chi_{\mathrm{Pf}}^{(\max )}, \chi_{\mathrm{Pa}}^{(\max )}$ and the integrated autocorrelation time of the fundamental and adjoint plaquettes $\tau_{\mathrm{f}}, \tau_{\mathrm{a}}$ for different adjoint couplings $\beta_{\text {adj }}$ and at fixed volume $L=20$. The statistical errors come from a jackknife procedure with bins of $3 \tau$ measures, while the error on $\beta_{\text {fund }}$ is estimated by the distance between neighbouring simulated points. The two lines at $\beta_{\text {adj }}=1.00$ are due to the fact that both $\beta_{\text {fund }}=$ 1.40 and $\beta_{\text {fund }}=1.41$ give compatible values for the measured maximum of the susceptibility, which indicates that the real maximum falls in between the two simulated $\beta_{\text {fund }}$. The starred point at $\beta_{\text {adj }}=1.00$ was used for the calculation of the spectrum at $\beta_{\text {adj }}=1.00$, while the other point at the same value of $\beta_{\text {adj }}$ was used for the susceptibility analyses. The fact that at fixed $\beta_{\text {adj }}$ different (close) values of $\beta_{\text {fund }}$ are suitable for a scaling analysis of different observables is due to the mildness of the crossover at the boundary of the critical region. 


\begin{tabular}{|c|c|c|c|c|}
\hline range $\beta_{\text {adj }}$ & $\beta_{\text {adj }}^{\text {(crit) }}$ & $\mathrm{A}$ & $\gamma$ & $\chi^{2} /$ dof \\
\hline $1.00-1.20$ & $1.2453(55)$ & $0.078(5)$ & $1.05(6)$ & 0.66 \\
$1.00-1.22$ & $1.2459(28)$ & $0.077(3)$ & $1.06(3)$ & 0.50 \\
$1.05-1.20$ & $1.2451(87)$ & $0.078(10)$ & $1.05(10)$ & 1.00 \\
$\mathbf{1 . 0 5}-\mathbf{1 . 2 2}$ & $\mathbf{1 . 2 4 6 0 ( 3 8 )}$ & $\mathbf{0 . 0 7 7 ( 5 )}$ & $\mathbf{1 . 0 6 ( 5 )}$ & $\mathbf{0 . 6 7}$ \\
$1.00-1.22^{*}$ & $1.2485(39)$ & $0.072(4)$ & $1.10(5)$ & 0.79 \\
\hline
\end{tabular}

Table 3. Fit results for $\chi_{\mathrm{Pf}}^{(\max )}$ according to the formula $\chi_{\mathrm{Pf}}^{(\max )}=A\left(\beta_{\mathrm{adj}}^{(\mathrm{crit})}-\beta_{\mathrm{adj}}\right)^{-\gamma}$. The starred value uses the starred point in table 2 . Boldfaced values are used in the text.

\begin{tabular}{|c|c|c|c|c|}
\hline range $\beta_{\text {fund }}$ & $\beta_{\text {fund }}^{\text {(crit) }}$ & $\mathrm{A}$ & $\gamma$ & $\chi^{2} /$ dof \\
\hline $1.241-1.40$ & $1.2206(34)$ & $0.053(5)$ & $1.09(6)$ & 1.69 \\
$\mathbf{1 . 2 4 1 - 1 . 3 7}$ & $\mathbf{1 . 2 2 2 9 ( 3 1 )}$ & $\mathbf{0 . 0 6 0 ( 6 )}$ & $\mathbf{1 . 0 3 ( 6 )}$ & $\mathbf{1 . 4 1}$ \\
$1.256-1.40$ & $1.2201(72)$ & $0.053(8)$ & $1.10(11)$ & 2.24 \\
$1.256-1.37$ & $1.2256(68)$ & $0.063(12)$ & $0.99(11)$ & 1.96 \\
$1.256-1.41^{*}$ & $1.2220(26)$ & $0.057(41)$ & $1.05(4)$ & 1.16 \\
\hline
\end{tabular}

Table 4. Fit results for $\chi_{\mathrm{Pf}}^{(\max )}$ according to the formula $\chi_{\mathrm{Pf}}^{(\max )}=A\left(\beta_{\text {fund }}-\beta_{\text {fund }}^{(\text {crit })}\right)^{-\gamma}$. The starred value uses the starred point in table 2 . Boldfaced values are used in the text.

\begin{tabular}{|c|c|c|c|c|c|c|}
\hline \multicolumn{7}{|c|}{$\beta_{\text {adj }}=1.00$} \\
\hline$\beta_{\text {fund }}$ & $L$ & $a m_{0^{++}}$ & $t_{i-t_{f}}$ & $L$ & $a \sqrt{\sigma}$ & $t_{i-} t_{f}$ \\
\hline 1.39 & 10 & $0.838(34)$ & $2-5$ & 10 & $0.4554(96)$ & $1-4$ \\
1.40 & 16 & $0.726(20)$ & $2-5$ & 10 & $0.3958(36)$ & $1-4$ \\
1.41 & 24 & $0.635(14)$ & $2-6$ & 16 & $0.3393(57)$ & $1-4$ \\
1.42 & 24 & $0.615(15)$ & $2-6$ & 24 & $0.2770(42)$ & $1-4$ \\
1.43 & 24 & $0.625(11)$ & $2-5$ & 24 & $0.2323(13)$ & $1-4$ \\
1.44 & 24 & $0.591(12)$ & $2-5$ & 24 & $0.1942(8)$ & $1-5$ \\
1.45 & 32 & $0.5781(77)$ & $1-6$ & 32 & $0.1635(15)$ & $2-6$ \\
1.46 & 32 & $0.4953(91)$ & $2-6$ & 32 & $0.1456(19)$ & $3-8$ \\
1.47 & 32 & $0.4593(88)$ & $2-6$ & 32 & $0.1263(14)$ & $3-10$ \\
\hline
\end{tabular}

Table 5. Values for $a m_{0^{++}}$and $a \sqrt{\sigma}$ on the lattices used as an estimate of the infinite volume limit for different $\beta_{\text {fund }}$ at $\beta_{\text {adj }}=1.00$. The time interval $t_{i} t_{f}$ indicates the fitting window used for the reported values. 


\begin{tabular}{|c|c|c|c|c|c|c|}
\hline \multicolumn{7}{|c|}{$\beta_{\text {adj }}=1.05$} \\
\hline$\beta_{\text {fund }}$ & $L$ & $a m_{0^{++}}$ & $t_{i}-t_{f}$ & $L$ & $a \sqrt{\sigma}$ & $t_{i^{-}} t_{f}$ \\
\hline 1.35 & 10 & $0.835(14)$ & $1-5$ & 6 & $0.466(10)^{*}$ & $1-4$ \\
1.36 & 10 & $0.699(23)$ & $1-7$ & 10 & $0.4177(47)$ & $1-4$ \\
1.37 & 16 & $0.578(14)$ & $2-6$ & 10 & $0.3413(50)$ & $2-5$ \\
1.38 & 16 & $0.567(14)$ & $2-6$ & 10 & $0.2588(15)$ & $2-6$ \\
1.39 & 24 & $0.579(13)$ & $2-7$ & 24 & $0.2098(28)$ & $2-6$ \\
1.40 & 24 & $0.569(12)$ & $2-6$ & 24 & $0.1737(11)$ & $2-5$ \\
1.41 & 32 & $0.4977(92)$ & $2-6$ & 32 & $0.1463(10)$ & $2-6$ \\
1.42 & 32 & $0.4414(77)$ & $2-6$ & 32 & $0.1265(7)$ & $2-6$ \\
1.43 & 32 & $0.3961(80)$ & $2-6$ & 32 & $0.1123(9)$ & $3-9$ \\
\hline
\end{tabular}

Table 6. Same as table 5 for $\beta_{\text {adj }}=1.05$. The stars ${ }^{*}$ indicate quantities for which only a single volume simulation is available.

\begin{tabular}{|c|c|c|c|c|c|c|}
\hline \multicolumn{7}{|c|}{$\beta_{\text {adj }}=1.10$} \\
\hline$\beta_{\text {fund }}$ & $L$ & $a m_{0^{++}}$ & $t_{i}-t_{f}$ & $L$ & $a \sqrt{\sigma}$ & $t_{i}-t_{f}$ \\
\hline 1.32 & 10 & $0.716(2)^{*}$ & $2-5$ & 10 & $0.4331(67)$ & $1-4$ \\
1.325 & 10 & $0.591(1)^{*}$ & $1-6$ & 10 & $0.3961(44)^{*}$ & $1-4$ \\
1.330 & 16 & $0.519(14)$ & $2-6$ & 10 & $0.3442(24)$ & $1-4$ \\
1.335 & 16 & $0.488(12)$ & $2-6$ & 16 & $0.2954(27)$ & $1-4$ \\
1.340 & 16 & $0.504(12)$ & $2-6$ & 16 & $0.2507(14)$ & $1-4$ \\
1.345 & 24 & $0.547(12)$ & $2-7$ & 24 & $0.2178(11)$ & $1-4$ \\
1.350 & 32 & $0.553(11)$ & $2-7$ & 24 & $0.1895(18)$ & $2-7$ \\
1.360 & 32 & $0.542(11)$ & $2-7$ & 32 & $0.1566(25)$ & $3-6$ \\
1.370 & 32 & $0.4555(92)$ & $2-6$ & 32 & $0.1275(7)$ & $2-6$ \\
\hline
\end{tabular}

Table 7. Same as table 5 for $\beta_{\text {adj }}=1.10$. The stars ${ }^{*}$ indicate quantities for which only a single volume simulation is available. 


\begin{tabular}{|c|c|c|c|c|c|c|}
\hline \multicolumn{7}{|c|}{$\beta_{\text {adj }}=1.16$} \\
\hline$\beta_{\text {fund }}$ & $L$ & $a m_{0^{++}}$ & $t_{i}-t_{f}$ & $L$ & $a \sqrt{\sigma}$ & $t_{i^{-}} t_{f}$ \\
\hline 1.275 & 16 & $0.679(10)^{*}$ & $1-5$ & - & - & - \\
1.28 & 16 & $0.5399(76)$ & $1-6$ & 10 & $0.417(21)$ & $2-5$ \\
1.285 & 24 & $0.4028(69)$ & $2-7$ & 12 & $0.3254(52)$ & $2-5$ \\
1.29 & 24 & $0.4192(66)$ & $2-7$ & 16 & $0.2485(30)$ & $2-5$ \\
1.295 & 24 & $0.4772(93)$ & $2-7$ & 24 & $0.2012(23)$ & $2-7$ \\
1.30 & 24 & $0.506(11)$ & $2-7$ & 24 & $0.1736(12)$ & $2-6$ \\
1.31 & 32 & $0.4612(85)$ & $2-6$ & 32 & $0.1348(8)$ & $2-8$ \\
1.32 & 32 & $0.4149(57)$ & $2-6$ & 32 & $0.1117(9)$ & $3-9$ \\
1.33 & - & - & - & 32 & $0.0958(5)$ & $3-9$ \\
\hline
\end{tabular}

Table 8. Same as table 5 for $\beta_{\text {adj }}=1.16$. The stars ${ }^{*}$ indicate quantities for which only a single volume simulation is available.

\begin{tabular}{|c|c|c|c|c|c|c|}
\hline \multicolumn{7}{|c|}{$\beta_{\text {adj }}=1.18$} \\
\hline$\beta_{\text {fund }}$ & $L$ & $a m_{0^{++}}$ & $t_{i}-t_{f}$ & $L$ & $a \sqrt{\sigma}$ & $t_{i}-t_{f}$ \\
\hline 1.2600 & 16 & $0.693(11)$ & $1-6$ & - & - & - \\
1.2625 & 16 & $0.645(16)$ & $2-5$ & - & - & - \\
1.2650 & 16 & $0.5213(73)$ & $1-6$ & - & - & - \\
1.2675 & 16 & $0.4458(93)$ & $2-6$ & 10 & $0.403(15)^{*}$ & $2-4$ \\
1.2700 & 32 & $0.3472(51)$ & $2-7$ & 10 & $0.3224(45)^{*}$ & $2-4$ \\
1.2710 & 32 & $0.3343(49)$ & $2-7$ & 12 & $0.3015(25)$ & $2-5$ \\
1.2725 & 24 & $0.3439(46)$ & $2-7$ & 12 & $0.2698(30)$ & $2-5$ \\
1.2750 & 24 & $0.3975(60)$ & $2-7$ & 24 & $0.2384(15)$ & $1-6$ \\
1.2775 & 24 & $0.4440(71)$ & $2-7$ & 24 & $0.2102(9)$ & $1-6$ \\
1.2800 & 24 & $0.4874(83)$ & $2-7$ & 24 & $0.1869(13)$ & $2-5$ \\
1.2825 & 24 & $0.5135(95)^{*}$ & $2-7$ & 24 & $0.1724(10)^{*}$ & $2-5$ \\
\hline
\end{tabular}

Table 9. Same as table 5 for $\beta_{\text {adj }}=1.18$. The stars ${ }^{*}$ indicate quantities for which only a single volume simulation is available. 


\begin{tabular}{|c|c|c|c|c|c|c|}
\hline \multicolumn{7}{|c|}{$\beta_{\text {adj }}=1.20$} \\
\hline$\beta_{\text {fund }}$ & $L$ & $a m_{0^{++}}$ & $t_{i}-t_{f}$ & $L$ & $a \sqrt{\sigma}$ & $t_{i-} t_{f}$ \\
\hline 1.254 & 16 & $0.3424(55)$ & $2-6$ & 10 & $0.3499(68)$ & $2-4$ \\
1.255 & 16 & $0.2963(43)$ & $2-7$ & 10 & $0.3245(49)$ & $2-4$ \\
1.256 & 48 & $0.2771(42)$ & $3-10$ & 12 & $0.2971(39)$ & $2-5$ \\
1.257 & 16 & $0.3008(49)$ & $2-7$ & 14 & $0.2676(32)$ & $2-6$ \\
1.258 & 16 & $0.3189(44)$ & $2-6$ & 16 & $0.2458(22)$ & $2-4$ \\
1.259 & 32 & $0.3595(54)$ & $2-8$ & 16 & $0.2290(18)$ & $2-5$ \\
1.261 & 32 & $0.4122(73)$ & $2-7$ & 32 & $0.2095(14)$ & $1-3$ \\
1.263 & 32 & $0.4522(76)$ & $2-7$ & 32 & $0.1896(9)$ & $1-4$ \\
\hline
\end{tabular}

Table 10. Same as table 5 for $\beta_{\mathrm{adj}}=1.20$.

\begin{tabular}{|c|c|c|c|c|c|c|c|}
\hline \multicolumn{5}{|c|}{$\beta_{\text {adj }}=1.00$} & \multicolumn{5}{|c|}{$\beta_{\text {adj }}=1.05$} \\
\hline$\beta_{\text {fund }}$ & $L$ & $a m_{2^{++}}$ & $t_{i}-t_{f}$ & $\beta_{\text {fund }}$ & $L$ & $a m_{2^{++}}$ & $t_{i}-t_{f}$ \\
\hline 1.39 & 10 & $2.56(37)$ & $1-3$ & 1.37 & 32 & $1.888(94)$ & $1-4$ \\
1.40 & 10 & $2.39(19)$ & $1-3$ & 1.38 & 16 & $1.455(49)^{*}$ & $1-4$ \\
1.41 & 16 & $1.92(11)$ & $1-5$ & 1.39 & 32 & $1.160(65)$ & $2-5$ \\
1.42 & 24 & $1.581(50)$ & $1-5$ & 1.40 & 24 & $0.910(29)^{*}$ & $2-5$ \\
1.43 & 24 & $1.237(26)$ & $1-4$ & 1.41 & 32 & $0.851(25)$ & $2-5$ \\
1.44 & 24 & $1.048(17)$ & $1-4$ & 1.42 & 32 & $0.666(16)$ & $2-6$ \\
1.45 & 32 & $0.909(31)$ & $2-6$ & - & - & - & - \\
1.46 & 32 & $0.732(20)$ & $2-6$ & - & - & - & - \\
1.47 & 32 & $0.687(14)$ & $2-6$ & - & - & - & - \\
\hline
\end{tabular}

Table 11. Values for $a m_{2^{++}}$on the lattices used as an estimate of the infinite volume limit for different $\beta_{\text {fund }}$ at $\beta_{\text {adj }}=1.00$ and $\beta_{\text {adj }}=1.05$. The time interval $t_{i}$ - $t_{f}$ indicates the fitting window used for the reported values. The stars * indicate quantities for which only a single volume simulation is available.

\begin{tabular}{|c|c|c|c|c|}
\hline range $\beta_{\text {adj }}$ & $\beta_{\text {adj }}^{\text {(crit) }}$ & $\mathrm{A}$ & $P$ & $\chi^{2} /$ dof \\
\hline $1.00-1.20$ & $1.2308(59)$ & $1.19(5)$ & $0.42(3)$ & 1.07 \\
$1.05-1.20$ & $1.2330(96)$ & $1.23(13)$ & $0.44(7)$ & 1.48 \\
$1.00-1.20$ & $1.2455(26)$ & $1.31(2)$ & 0.5 & 1.97 \\
$1.05-1.20$ & $1.2420(28)$ & $1.36(3)$ & 0.5 & 1.37 \\
\hline
\end{tabular}

Table 12. Fit results for $a m_{0^{++}}$according to the formula $a m_{0^{++}}=A\left(\beta_{\text {adj }}^{\text {(crit) }}-\beta_{\text {adj }}\right)^{P}$. In the last two lines, $P$ is kept fixed to 0.5 . 


\begin{tabular}{|c|c|c|c|c|}
\hline range $\beta_{\text {fund }}$ & $\beta_{\text {fund }}^{\text {(crit) }}$ & $\mathrm{A}$ & $P$ & $\chi^{2} /$ dof \\
\hline $1.256-1.41$ & $1.2346(47)$ & $1.30(8)$ & $0.40(4)$ & 1.64 \\
$1.256-1.37$ & $1.2326(77)$ & $1.37(20)$ & $0.43(7)$ & 2.23 \\
$1.256-1.41$ & $1.2210(25)$ & $1.50(3)$ & 0.5 & 3.14 \\
$1.256-1.37$ & $1.2246(26)$ & $1.57(5)$ & 0.5 & 2.10 \\
\hline
\end{tabular}

Table 13. Fit results for for $a m_{0^{++}}$according to the formula $a m_{0^{++}}=A\left(\beta_{\text {fund }}-\beta_{\text {fund }}^{(\text {crit })}\right)^{P}$. In the last two lines, $P$ is kept fixed to 0.5 .

\begin{tabular}{|c|c|c|}
\hline method & $\beta_{\mathrm{adj}}^{\text {(crit) }}$ & $\beta_{\text {fund }}^{\text {(crit) }}$ \\
\hline latent heat (eq. (3.1)) & $1.22-1.25$ & - \\
$\chi_{\mathrm{Pf}}^{(\max )}$ scaling (eq. (3.2)) & $1.2460(38)$ & $1.2229(31)$ \\
$a m_{0^{++}}$scaling (eq. (5.1)) & $1.2308(59)$ & $1.2346(47)$ \\
with fixed exponent $(P=0.5)$ & $1.2455(26)$ & $1.2210(25)$ \\
\hline
\end{tabular}

Table 14. Summary of the different estimates of the critical $\left(\beta_{\text {fund }}, \beta_{\text {adj }}\right)$ values described in the text.

\section{References}

[1] ATLAS collaboration, Observation of a new particle in the search for the standard model Higgs boson with the ATLAS detector at the LHC, Phys. Lett. B 716 (2012) 1 [arXiv: 1207.7214] [INSPIRE].

[2] CMS collaboration, Observation of a new boson at a mass of $125 \mathrm{GeV}$ with the CMS experiment at the LHC, Phys. Lett. B 716 (2012) 30 [arXiv:1207.7235] [INSPIRE].

[3] S. Weinberg, Implications of dynamical symmetry breaking, Phys. Rev. D 13 (1976) 974 [INSPIRE].

[4] L. Susskind, Dynamics of spontaneous symmetry breaking in the Weinberg-Salam theory, Phys. Rev. D 20 (1979) 2619 [INSPIRE].

[5] B. Holdom, Techniodor, Phys. Lett. B 150 (1985) 301 [INSPIRE].

[6] K. Yamawaki, M. Bando and K.-i. Matumoto, Scale invariant technicolor model and a technidilaton, Phys. Rev. Lett. 56 (1986) 1335 [InSPIRE].

[7] T.W. Appelquist, D. Karabali and L.C.R. Wijewardhana, Chiral hierarchies and the flavor changing neutral current problem in technicolor, Phys. Rev. Lett. 57 (1986) 957 [INSPIRE].

[8] L. Del Debbio, The conformal window on the lattice, PoS(LATTICE 2010) 004.

[9] E.T. Neil, Exploring models for new physics on the lattice, PoS (LATTICE 2011) 009 [arXiv: 1205.4706] [INSPIRE].

[10] J. Giedt, Lattice gauge theory and physics beyond the standard model, PoS (LATTICE 2012) 006.

[11] D.D. Dietrich and F. Sannino, Walking in the $\mathrm{SU}(N)$, hep-ph/0611341 [INSPIRE].

[12] D.D. Dietrich and F. Sannino, Conformal window of $\mathrm{SU}(N)$ gauge theories with fermions in higher dimensional representations, Phys. Rev. D 75 (2007) 085018 [INSPIRE]. 
[13] L. Del Debbio, B. Lucini, A. Patella, C. Pica and A. Rago, Conformal versus confining scenario in SU(2) with adjoint fermions, Phys. Rev. D 80 (2009) 074507 [arXiv:0907.3896] [INSPIRE].

[14] S. Catterall, J. Giedt, F. Sannino and J. Schneible, Probes of nearly conformal behavior in lattice simulations of minimal walking technicolor, arXiv:0910.4387 [INSPIRE].

[15] B. Lucini, Strongly interacting dynamics beyond the standard model on a spacetime lattice, Phil. Trans. Roy. Soc. Lond. A 368 (2010) 3657 [arXiv:0911.0020] [INSPIRE].

[16] L. Del Debbio, B. Lucini, A. Patella, C. Pica and A. Rago, The infrared dynamics of minimal walking technicolor, Phys. Rev. D 82 (2010) 014510 [arXiv:1004.3206] [INSPIRE].

[17] L. Del Debbio, B. Lucini, A. Patella, C. Pica and A. Rago, Mesonic spectroscopy of minimal walking technicolor, Phys. Rev. D 82 (2010) 014509 [arXiv: 1004.3197] [InSPIRE].

[18] A.J. Hietanen, K. Rummukainen and K. Tuominen, Evolution of the coupling constant in SU(2) lattice gauge theory with two adjoint fermions, Phys. Rev. D 80 (2009) 094504 [arXiv: 0904.0864] [INSPIRE].

[19] F. Bursa, L. Del Debbio, L. Keegan, C. Pica and T. Pickup, Mass anomalous dimension in SU(2) with two adjoint fermions, Phys. Rev. D 81 (2010) 014505 [arXiv:0910.4535] [INSPIRE].

[20] T. DeGrand, Y. Shamir and B. Svetitsky, Infrared fixed point in SU(2) gauge theory with adjoint fermions, Phys. Rev. D 83 (2011) 074507 [arXiv:1102.2843] [InSPIRE].

[21] S. Catterall, L. Del Debbio, J. Giedt and L. Keegan, MCRG minimal walking technicolor, Phys. Rev. D 85 (2012) 094501 [arXiv:1108.3794] [InSPIRE].

[22] S. Catterall and F. Sannino, Minimal walking on the lattice, Phys. Rev. D 76 (2007) 034504 [arXiv:0705.1664] [INSPIRE].

[23] L. Del Debbio, A. Patella and C. Pica, Higher representations on the lattice: Numerical simulations. SU(2) with adjoint fermions, Phys. Rev. D 81 (2010) 094503 [arXiv: 0805.2058] [INSPIRE].

[24] A. Hietanen, J. Rantaharju, K. Rummukainen and K. Tuominen, Minimal technicolor on the lattice, Nucl. Phys. A 820 (2009) 191C [inSPIRE].

[25] F. Bursa et al., Improved lattice spectroscopy of minimal walking technicolor, Phys. Rev. D 84 (2011) 034506 [arXiv:1104.4301] [INSPIRE].

[26] T. Karavirta, A. Mykkanen, J. Rantaharju, K. Rummukainen and K. Tuominen, Nonperturbative improvement of $\mathrm{SU}(2)$ lattice gauge theory with adjoint or fundamental flavors, JHEP 06 (2011) 061 [arXiv:1101.0154] [INSPIRE].

[27] A. Patella, L. Del Debbio, B. Lucini, C. Pica and A. Rago, Finite volume effects in SU(2) with two adjoint fermions, PoS (LATTICE 2011) 084 [arXiv:1111.4672] [INSPIRE].

[28] E. Bennett and B. Lucini, Topology of minimal walking technicolor, Eur. Phys. J. C 73 (2013) 2426 [arXiv: 1209.5579] [INSPIRE].

[29] J. Giedt and E. Weinberg, Finite size scaling in minimal walking technicolor, Phys. Rev. D 85 (2012) 097503 [arXiv: 1201.6262] [INSPIRE].

[30] A. Patella, A precise determination of the $\bar{\psi}-\psi$ anomalous dimension in conformal gauge theories, Phys. Rev. D 86 (2012) 025006 [arXiv:1204.4432] [InSPIRE].

[31] G. Bhanot and M. Creutz, Variant actions and phase structure in lattice gauge theory, Phys. Rev. D 24 (1981) 3212 [INSPIRE]. 
[32] R.V. Gavai, M. Grady and M. Mathur, Finite temperature phase transition in $\mathrm{SU}(2)$ lattice gauge theory with extended action, Nucl. Phys. B 423 (1994) 123 [hep-lat/9403001] [INSPIRE].

[33] M. Mathur and R.V. Gavai, Universality and the deconfinement phase transition in $\mathrm{SU}(2)$ lattice gauge theory, Nucl. Phys. B 448 (1995) 399 [hep-lat/9410004] [INSPIRE].

[34] P. Stephenson, Physical and unphysical effects in the mixed $\mathrm{SU}(2) / \mathrm{SO}(3)$ gauge theory, hep-lat/9604008 [INSPIRE].

[35] R.V. Gavai, A Study of the bulk phase transitions of the SU(2) lattice gauge theory with mixed action, Nucl. Phys. B 474 (1996) 446 [hep-lat/9603003] [INSPIRE].

[36] S. Datta and R.V. Gavai, Stability of the bulk phase diagram of the SU(2) lattice gauge theory with fundamental adjoint action, Phys. Lett. B 392 (1997) 172 [hep-lat/9610022] [INSPIRE].

[37] M. Grady, Critical or tricritical point in mixed-action $\mathrm{SU}(2)$ lattice gauge theory?, Nucl. Phys. B 713 (2005) 204 [hep-lat/0404015] [INSPIRE].

[38] E. Rinaldi, G. Lacagnina, B. Lucini, A. Patella and A. Rago, Scaling properties of SU(2) gauge theory with mixed fundamental-adjoint action, PoS (LATTICE 2012) 252 [arXiv: 1212.0635] [INSPIRE].

[39] L. Caneschi, I. Halliday and A. Schwimmer, The phase structure of mixed lattice gauge theories, Nucl. Phys. B 200 (1982) 409 [InSPIRE].

[40] U.M. Heller, SU(3) lattice gauge theory in the fundamental adjoint plane and scaling along the Wilson axis, Phys. Lett. B 362 (1995) 123 [hep-lat/9508009] [INSPIRE].

[41] A. Bazavov, B.A. Berg and U.M. Heller, Biased metropolis-heat-bath algorithm for fundamental-adjoint SU(2) lattice gauge theory, Phys. Rev. D 72 (2005) 117501 [hep-lat/0510108] [INSPIRE].

[42] A. Bazavov and B.A. Berg, Heat bath efficiency with metropolis-type updating, Phys. Rev. D 71 (2005) 114506 [hep-lat/0503006] [INSPIRE].

[43] H. Vairinhos, Monte Carlo algorithms for reduced lattices, mixed actions, and double-trace deformations, arXiv:1010.1253 [INSPIRE].

[44] B. Lucini, A. Rago and E. Rinaldi, Glueball masses in the large-N limit, JHEP 08 (2010) 119 [arXiv: 1007.3879] [INSPIRE].

[45] B. Lucini and $\mathrm{M}$. Teper, The $k=2$ string tension in four dimensional $\mathrm{SU}(N)$ gauge theories, Phys. Lett. B 501 (2001) 128 [hep-lat/0012025] [INSPIRE]. 